\title{
Structural changes of laminar separation bubbles induced by global linear instability
}

\author{
D. RODRÍGUEZ and V. THEOFILIS $\dagger$ \\ School of Aeronautics. Universidad Politécnica de Madrid, Pza. Cardenal Cisneros 3, \\ E-28040 Madrid, Spain
}

The topology of the composite flow fields reconstructed by linear superposition of a two-dimensional boundary layer flow with an embedded laminar separation bubble and its leading three-dimensional global eigenmodes has been studied. According to critical point theory, the basic flow is structurally unstable; it is shown that in the presence of three-dimensional disturbances the degenerate basic flow topology is replaced by a fully three-dimensional pattern, regardless of the amplitude of the superposed linear perturbations. Attention has been focused on the leading stationary eigenmode of the laminar separation bubble discovered by Theofilis et al. (Phil. Trans. R. Soc. Lond. A, vol. 358, 2000, pp. 3229-3324); the composite flow fields have been fully characterized with respect to the generation and evolution of their critical points. The stationary global mode is shown to give rise to a three-dimensional flow field which is equivalent to the classical U-shaped separation, defined by Hornung \& Perry (Z. Flugwiss. Weltraumforsch., vol. 8, 1984, pp. 77-87), and induces topologies on the surface streamlines that are resemblant to the characteristic stall cells observed experimentally.

\section{Introduction}

The instability of a laminar separation bubble (LSB), embedded inside a boundary layer and arising as a consequence of an adverse free-stream pressure gradient on a flat surface, or induced by changes of the surface curvature, constitutes a problem of prime technological significance: in an aeronautical context related applications include lifting surfaces, low-pressure turbines and wind-turbine blades. The presence of a separated shear layer as an integral part of the LSB suggests that the instability properties of the shear layer should play a decisive role in the reattachment of the separated boundary layer. The one-dimensionality of the model shear-layer profile and its resulting amenability to classic, ordinary-differential-equation-based analysis (Michalke 1964; Blumen 1970 and subsequent work in this 'local' vein) has generated a wealth of knowledge on the problem of shear-layer instability per-se and as applied to the LSB flow. On the other hand, Gaster postulated (H. Fasel, private communication November 2004) that, besides paying attention to the amplification of incoming disturbances by solution of the local instability problem, analysis of LSB flows 'in the true sense' should also be performed, by which a global instability analysis of the entire separated boundary layer flow is implied. 
In the latter context Allen \& Riley (1995) employed absolute/convective instability analysis (Huerre \& Monkewitz 1985, 1990) on two different classes of separated basic flows, namely a backward-facing slope and a Joukowski airfoil at different Reynolds numbers. In all cases analysed, they found a strong convective instability, but no evidence of absolute instability. On the other hand. Hammond \& Redekopp (1998) also employed absolute/convective instability analysis to study perturbations in a model of LSB flow, the latter constructed using similarity solutions. These authors determined that absolute instability was present when the peak reversed flow amplitude, scaled with the far-field velocity, exceeds $\sim 20 \%$. Independent investigations using linear instability theory and/or direct numerical simulations (DNS) (Rist \& Maucher 2002; Fasel \& Postl 2004) agree reasonably well with this threshold for the onset of absolute instability of wave-like disturbances of the LSB. However, it was Theofilis (2000) and Theofilis, Hein \& Dallmann (2000) who first unequivocally demonstrated the self-excitation potential of LSB flows, by solving the partial-derivative-based eigenvalue problem pertinent to the (twodimensional DNS obtained) separated boundary layer ensuing Howarth's freestream linear deceleration on a flat plate (Briley 1971), without the need to invoke the assumption of weak non-parallelism of the basic state; at about the same time, an analogous conclusion was independently reached by Barkley, Gomes \& Henderson (2002) in the LSB formed in the laminar backward-facing step flow.

In the last decade instability analyses based on solutions of partial-derivative eigenvalue problems (often referred to as BiGlobal analyses, Theofilis 2003) and/or DNS have elucidated global instability mechanisms in a variety of LSB flows, including the aforementioned adverse-pressure-gradient flat-plate flow (Theofilis et al. 2000), backward-facing step flow (Barkley et al. 2002), as well as geometry-induced separation (Marquillie \& Ehrenstein 2003; Gallaire, Marquillie \& Ehrenstein 2007). NACA 0012 airfoil in incompressible (Theofilis, Barkley \& Sherwin 2002) and compressible flow (Crouch, Garbaruk \& Magidov 2007), low-pressure-turbine flow (Abdessemed, Sherwin \& Theofilis 2004, 2009), shock-induced separation (Boin et al. 2006; Robinet 2007), open cavity (Åkervik et al. 2007) and S-shaped duct flows (Marquet $e$ al. 2008, 2009). In all configurations examined consensus has been built that two different primary instability mechanisms coexist. External disturbances, like Tollmien-Schlichting (TS) waves, entering the bubble are highly amplified by the shear layer of the bubble, and then the classical Kelvin-Helmholtz $(\mathrm{KH})$ mechanism is recovered. In addition, LSB flows have the potential to self-excite an intrinsic instability mechanism, referred to as the global mode of the bubble, the main characteristics of which are its stationary and three-dimensional nature (Theofilis et al. 2000).

The present contribution revisits a BiGlobal analysis of instability of LSB flows on a flat plate. The substantial increase in computing hardware capabilities in the last decade, in conjunction with the recent development of massively parallel numerical solutions of the partial-derivative eigenvalue problem (Rodríguez \& Theofilis 2009), permit utilization of orders-of-magnitude higher resolution compared with that employed a decade ago for the solution of the same problem. Although some reference is made to the conditions under which self-sustained global instability may appear in this LSB flow, as well as to the dependence of the global amplification rates on the parameters defining the LSB flow, the focus of the present work is on application of critical-point theory to the analysis of the topological bifurcations exerted by the intrinsic global mode of laminar separation. 
Critical point concepts emerged in the context of fluid flow in the early eighties of last century and have since become a powerful tool in describing exact local solutions of the equations of motion; such local solutions may then be used in order to reconstruct the entire (laminar or turbulent) three-dimensional flow field the interested reader is referred to Dallmann (1982), Hornung \& Perry (1984), Perry \& Hornung (1984), Perry \& Chong (1987) and Dallmann, Vollmers \& Su (1997) for the fundamentals of the reconstruction and classification of separated flow topologies and, for example, to Vorobieff \& Rockwell (1996) for one application of the theory to a concrete flow control problem. According to critical point theory, the two-dimensional flow structure of the laminar separated basic field is described by means of centre and node-saddle points. This topology is defined as a degeneracy, which is only possible in strictly two-dimensional flows. The small three-dimensional disturbances resulting from linear analysis will alter the flow topology, independently of the amplitudes considered, and fully three-dimensional patterns will appear. The objective of the present work is to investigate the topological changes exerted by the global mode over the LSB. Unlike Theofilis et al. (2000) where an analogous discussion was qualitative and based on theoretical arguments presented in earlier works (Dallmann et al. 1997), the reconstructions of the flow field considered here are quantitative, and are based on linear superposition of the eigenmodes obtained from the present global linear instability analysis upon the respective basic state.

The paper is organized as follows: in $\$ 2$ the LSB basic flow analysed is described. $\$ 3$ is devoted to the BiGlobal linear instability analysis. Direct numerical simulations, performed in order to verify the results of the instability analysis, are presented in $\$ 4$. The main points of the critical-point theory and its application to the topology of the disturbed LSB are exposed in $\$ 5$. A discussion of the results and qualitative comparison with experimental work in $\$ 6$ closes the present contribution.

\section{Basic flow construction}

\subsection{Inverse formulation of the non-similar boundary-layer problem}

A non-similar inverse formulation of the boundary-layer equations on a flat plate was used to obtain the basic flow. Two-dimensional DNS of the Navier-Stokes equations used in earlier work (Briley 1971; Theofilis et al. 2000), in which a linear deceleration of the far-field streamwise velocity was imposed in order to force the appearance of the LSB, were dismissed in the present context. In choosing the current nonsimilar boundary-layer formulation, the appearance of the bubble shedding as the peak reversed flow increases is circumvented in the computation of the basic flow and may be subsequently recovered as one (or more) global eigenmodes of this basic flow.

The physical dimensional streamwise, wall-normal and spanwise coordinates are denoted as $x^{*}, y^{*}$ and $z^{*}$, respectively; the far-field velocity is $U_{e}^{*}$, the streamfunction is $\Psi$ and the kinematic viscosity is $\nu$. The non-dimensional boundary-layer variables

$$
\xi=x^{*} / L, \quad \eta=y^{*} \sqrt{U_{e}^{*} / v x^{*}},
$$

and transformed streamfunction

$$
f(\xi, \eta)=\Psi / \sqrt{U_{e}^{*} v x^{*}}
$$

are introduced into the streamwise momentum equation in order to obtain an equation in the transformed streamfunction $f(\xi, \eta)$. The streamwise length $L$ is an arbitrary quantity used to make $x^{*}$ dimensionless. In order to recover separated states, the 
Reyhner and Flïgge-Lotz (FLARE) approximation (Cebeci \& Cousteix 2001) has been invoked, which neglects the streamwise convective term when reversed flow exists. The boundary-layer equation is written as

$$
f_{\eta \eta \eta}+\frac{m+1}{2} f f_{m}+m\left(1-f_{\eta}^{2}\right)=\xi\left(\theta f_{\eta} f_{\xi \eta}+f_{m} f_{\xi}\right),
$$

where subscripts imply partial differentiation, and $m=\left(\xi / U_{e}^{*}\right)\left(\mathrm{d} U_{e}^{*} / \mathrm{d} \xi\right)$ is the deceleration parameter, which is variable with $\xi$. The FLARE approximation appears in this equation as the function

$$
\theta= \begin{cases}1, & f_{\eta} \geqslant 0 . \\ 0, & f_{\eta}<0 .\end{cases}
$$

This problem is solved subjected to the boundary conditions

$$
f(\xi, 0)=0, \quad f_{\eta}(\xi, 0)=0 \text { and } f_{\eta}(\xi, \eta \rightarrow \infty) \rightarrow 1 .
$$

If (2.3) were solved in a direct context, the far-field deceleration would be fixed and the distribution $m(\xi)$ would be imposed, which would lead to the well-known Goldstein singularity at separation. On the other hand, inverse formulations, such as the one used herein, in which the wall shear or the displacement thickness are imposed, can recover separated states. Here, the displacement thickness measured in the transformed variable $\eta$ is imposed as the boundary condition

$$
f(\xi, \eta \rightarrow \infty) \rightarrow 1-\bar{\delta}(\xi) .
$$

The complete derivation of the equation and boundary conditions can be found in Cebeci \& Cousteix (2001), while further details on the solution procedure have been presented by Rodriguez \& Theofilis (2008).

\subsection{The boundary-layer-based LSB model basic flow}

The two-dimensional LSB basic state $\bar{q}$, appearing in (3.1) in what follows, is constructed using the calculated transformed streamfunction $f(\xi, \eta)$ (defined in (2.2)). The streamwise and wall-normal dimensional velocity components are obtained using the transformations (2.1) and (2.2):

$$
\begin{aligned}
u^{*} & =U_{e}^{*} \cdot f_{\eta}, \\
v^{*} & =\frac{1}{2} \sqrt{\frac{U_{e}^{*} v}{\xi}}\left[f(m+1)+2 \xi f_{\xi}+\eta f_{\eta}(m-1)\right] .
\end{aligned}
$$

Lengths here are scaled using the dimensional displacement thickness at the inflow boundary $\delta_{i n}^{*}$, while the physical streamwise and wall-normal velocity components $u^{*}$ and $v^{*}$, and their first derivatives are made dimensionless using the inflow far-field velocity, $U_{e}^{*}$ and $\delta_{i n}^{*}$. The non-dimensional spatial variables are denoted by $x$ and $y$ and the dimensionless velocities by $\bar{u}$ and $\bar{v}$. These velocity components, along with their first spatial derivatives, constitute the basic flow for the BiGlobal instability analysis presented in $\$ 3$. Note that the basic flow is two-dimensional and the spanwise velocity $\bar{w}=0$.

The domain on the flat plate is chosen such that the Reynolds number based on this displacement thickness at inflow is $R e_{i n}=450$, and that at the outflow is $R e_{o u t}=700$. The domain box is $\Omega=L_{x} \times L_{y}=(152,370) \times(0,40)$. A larger domain box was also computed in order to accommodate a fringe region at the outflow for the DNS, as explained in $\$ 4$. 


\begin{tabular}{lccccccc}
\hline $\bar{\delta}_{\max }$ & $x_{\text {sep }}$ & $x_{\text {reat }}$ & $L_{L S B}$ & $\tau_{\operatorname{mir}}$ & $x_{\tau_{\text {taln }}}$ & $u_{\text {rev }}$ & $\left(\omega_{i}\right)_{\max } \times 10^{3}$ \\
3.25 & 229.9 & 255.0 & 25.1 & -0.061 & 247.2 & 1.11 & -0.136 \\
3.5 & 228.1 & 257.1 & 29.0 & -0.076 & 249.9 & 1.70 & -0.102 \\
4 & 226.1 & 260.0 & 33.9 & -1.063 & 253.8 & 2.78 & -4.938 \\
4.5 & 224.6 & 261.9 & 37.3 & -1.375 & 256.6 & 3.73 & -2.691 \\
5 & 223.7 & 263.1 & 39.4 & -1.701 & 258.7 & 4.57 & -1.480 \\
6 & 223.2 & 264.7 & 41.5 & -2.385 & 261.3 & 6.00 & -2.411 \\
7 & 221.6 & 265.8 & 44.2 & -3.094 & 262.8 & 7.16 & +3.439 \\
8 & 221.0 & 266.3 & 45.3 & -3.854 & 263.9 & 8.14 & +6.791
\end{tabular}

TABLE 1. Characteristic parameters of the laminar separation bubble models.

An analytical displacement thickness distribution analogous to that prescribed by Carter (1975) is used for (2.6), in which $\bar{\delta}$ starts at the Blasius value at the inflow boundary, is increased within a finite $x$-range until a maximum thickness $\bar{\delta}_{\max }$ is attained, is then decreased until the Blasius solution is again recovered and is maintained constant until the outflow boundary:

$$
\bar{\delta}(x)=\left(\bar{\delta}_{\max }-\bar{\delta}_{B}\right) \cdot\left[S\left(\xi_{1}\right)-S\left(\xi_{2}\right)\right]+\bar{\delta}_{B}
$$

where

$$
S(\xi)=\left\{\begin{array}{l}
0, \quad \xi<0, \\
{\left[1+\exp \left(\frac{1}{\xi-1}+\frac{1}{\xi}\right)\right]^{-1}, \quad 0 \leqslant \xi \leqslant 1,} \\
1, \quad 1<\xi,
\end{array}\right.
$$

and the auxiliary non-dimensional coordinates $\xi_{1}$ and $\xi_{2}$ are defined as

$$
\xi_{1}=a \frac{2(x-210)}{264-210}, \quad \xi_{2}=a \frac{2 x-210-264}{264-210} .
$$

The coordinates $x=210$ and $x=264$ define, respectively, the locations where the increase of displacement thickness starts and ends. The parameter $(a)$ is used to determine the maximum slope of $S$, and was fixed at $a=0.65$. While delivering displacement thickness distributions which are analogous to the piecewise polynomial distributions used in Carter (1975) and Rodriguez \& Theofilis (2008), the present function has the advantage of continuous derivatives of all orders. These distributions are a variation of the typical fringe function used in the formulation of DNS proposed by Lundbladh et al. (1994). The value $\bar{\delta}_{\max }$ acts as a parameter defining the strength of the LSB in terms of both wall-normal extension and reversed flow, and $\bar{\delta}_{B}=1.72078$ is the displacement thickness corresponding to the Blasius velocity profile. As a result of this choice of the parameter $(a)$, the actual maximum displacement thickness of the bubble is $\delta_{\max } \approx 0.96 \times \bar{\delta}_{\max }$.

Table 1 summarizes the characteristic parameters for the series of basic flows considered: the parameters appearing are the maximum displacement thickness of the model $\bar{\delta}_{\max }$, the primary separation location $x_{\text {sep }}$, the primary reattachment location $x_{\text {reat }}$, the streamwise length of the basic bubble $L_{L S B}$, the peak scaled wall shear $\tau=f_{\eta \eta}$, the location of the peak wall shear $x_{\tau_{m i n}}$ and the peak reversed flow, scaled with the inflow free-stream velocity $u_{r e v}=u_{r e v}^{*} / U_{e}^{*}$. The last column shows the maximum amplification rate predicted by the BiGlobal analysis for each case, as will be discussed in $\$ 3$. 

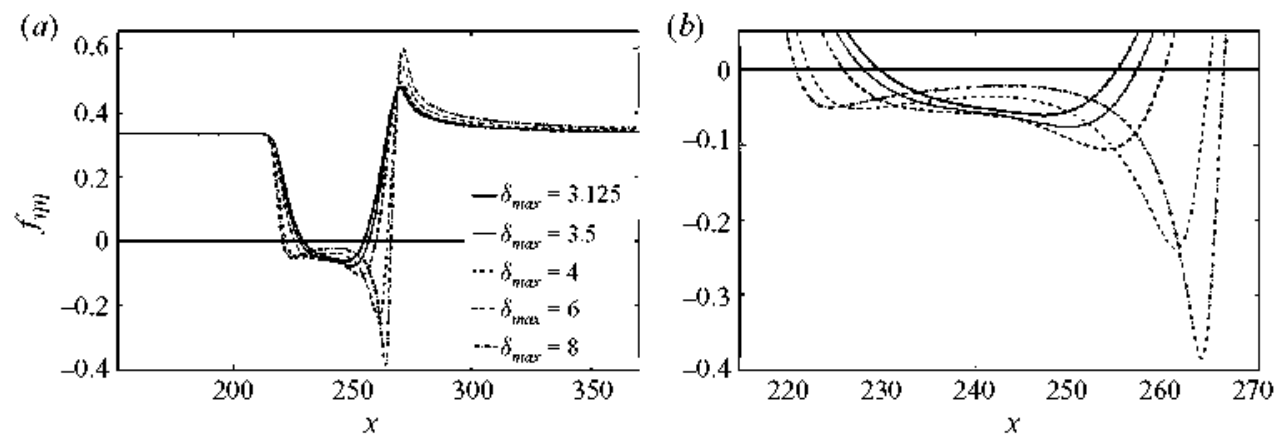

FIGURE 1. (a) Transformed wall-shear $\left(\tau=f_{m m}\right)$ distributions for five LSBs.

(b) The zoomed separated region.

The distributions of wall shear for five representative cases are shown in figure 1. In the cases where $\bar{\delta}_{\max }$ is low the wall-shear distribution within the separated region is nearly symmetric, attaining the minimum value near the midpoint between separation and reattachment. The location of the peak wall shear is related to the location of the centre of the recirculation region. As $\bar{\delta}_{\max }$ is increased the peak wall shear is increased and displaced downstream, and therefore the vortex centre moves downstream.

A peak of wall shear appears in the separated region, the magnitude of which is also increased with the size of the bubble. This evolution defines topological changes of the basic LSBs that can be explained as the appearance of an inflection point in the wall-shear distribution. As will be shown later, the topology of the basic LSB is found to have an important effect in the instability characteristics of the global mode. Also is worth of mention the overshoot in the recovery of the zero-pressure-gradient wall shear. This overshoot is a typical issue of inverse formulations of the boundary layer problem (Carter 1975) and formulations considering viscous-inviscid iteration (Cebeci, Keller \& Williams 1979; Allen \& Riley 1995).

\section{Direct and adjoint BiGlobal instability analyses}

\subsection{The BiGlobal eigenvalue problems}

The equations governing the two-dimensional eigenvalue problems solved are presented here for completeness. A solution $q(x, y, z, t)=[u, v, w, p]^{T}$ to the incompressible equations of motion is decomposed into

$$
\boldsymbol{q}(x, y, z, t)=\overline{\boldsymbol{q}}(x, y)+\varepsilon \hat{\boldsymbol{q}}(x, y) \exp (\mathrm{i} \Theta)+c . c .
$$

where $\Theta=\beta z-\omega t, \varepsilon \ll 1, \bar{q}$ indicates the basic flow calculated in $\S 2, \hat{q}$ indicates modal disturbances, $c . c$. denotes complex conjugate, $\beta$ is a real wavenumber related to the spanwise periodicity length through $L_{3}=2 \pi / \beta$ and $\omega$ is the complex eigenvalue sought. The real part of the eigenvalue, $\omega_{r}$, is the circular frequency of the eigenmode, while the imaginary part, $\omega_{i}$, is its temporal amplification rate. The decomposition is introduced into the incompressible continuity and Navier-Stokes equations. Linearization about the basic flow follows, based on the smallness of $\varepsilon$, and the basic flow terms are subtracted out, as they satisfy the equations of motion to the degree discussed in the previous section; further verification of the basic flow model will be provided in $\S 4$, which deals with the DNS work. The following system 
of equations for the determination of $\hat{q}$ results:

$$
\begin{aligned}
& \mathscr{Q}_{x} \hat{u}+\mathscr{O}_{y} \hat{v}+\mathrm{i} \hat{w}=0 \text {. } \\
& {\left[\mathscr{L}-\left(\mathscr{D}_{x} \bar{u}\right)\right] \hat{u}-\left(\mathscr{Q}_{y} \bar{u}\right) \hat{v}-\mathscr{P}_{x} \hat{p}=-\mathrm{i} \omega \hat{u},} \\
& -\left(\mathscr{Q}_{x} \bar{v}\right) \hat{u}+\left[\mathscr{Z}-\left(\mathscr{Q}_{y} \bar{v}\right)\right] \hat{v}-\mathscr{Q}_{y} \hat{p}=-\mathbf{i} \omega \hat{v}, \\
& \mathscr{L} \hat{\mathrm{w}}-\mathrm{i} \beta \hat{p}=-\mathrm{i} \omega \hat{\mathrm{w}},
\end{aligned}
$$

where $\mathscr{L}=(1 / \operatorname{Re})\left(\bar{L}_{x}^{2}+\mathscr{P}_{y}^{2}-\beta^{2}\right)-\bar{u} \overline{2} x-\bar{v} \mathscr{P}_{y}, \mathscr{D}_{x}=\partial / \partial x$, and $\mathscr{P}_{y}=\partial / \partial y$. The system of equations (3.2)-(3.5), complemented by the boundary conditions discussed below, constitutes an eigenvalue problem for the complex eigenvalue $\omega$, defined in the twodimensional domain $\Omega$.

The non-normality of the linearized Navier-Stokes equations results in the nonnormality of the eigenfunction basis obtained as solution of the (direct) eigenvalue problem (3.2)-(3.5). If an eigenfunction expansion of the disturbance field is sought, the definition of the adjoint problem is necessary in order to introduce a biorthogonality condition between the different eigenmodes (Morse \& Feshbach 1953; Salwen \& Grosch 1981; Tumin 2003). Applying the usual inner product for complex functions

$$
\langle\boldsymbol{a}, \boldsymbol{b}\rangle=\int_{\Omega} \boldsymbol{a}^{H} \cdot \boldsymbol{b} \mathrm{d} \Omega
$$

where the superseript denotes transposed complex-conjugate to the linear problem $\mathscr{N}(\bar{q}) \cdot \hat{q}=0$ defined by (3.2)-(3.5), the following Green's identity (Morse \& Feshbach $1953)$ is built:

$$
\langle\tilde{\boldsymbol{q}}, \hat{\mathcal{H}}(\overline{\boldsymbol{q}}) \cdot \hat{\boldsymbol{q}}\rangle=[\langle\tilde{\boldsymbol{v}}, \hat{\boldsymbol{v}}\rangle]_{0}^{t}+[\boldsymbol{J}(\tilde{\boldsymbol{q}}, \hat{\boldsymbol{q}})]_{\partial \Omega}-\left\langle\boldsymbol{N}^{\dagger}(\overline{\boldsymbol{q}}) \cdot \tilde{\boldsymbol{q}}, \hat{\boldsymbol{q}}\right\rangle .
$$

The term $N^{\dagger}(\bar{q}) \cdot \check{q}=0$ inside the inner product at the right-hand side defines the adjoint problem, where $\tilde{q}=(\tilde{u}, \tilde{v}, \tilde{w}, \tilde{p})^{T}$ is the vector of adjoint variables. The adjoint operator $\mathscr{W}^{\dagger}(\bar{q})$ is derived by integration by parts, and yields the adjoint eigenvalue problem

$$
\begin{gathered}
\mathscr{L}_{x} \tilde{u}+\mathscr{B}_{y} \tilde{v}-\mathrm{i} \beta \tilde{w}=0, \\
{\left[\mathscr{L}^{\dagger}-\left(\mathscr{D}_{x} \bar{u}\right)\right] \tilde{u}-\left(\mathscr{V}_{x} \bar{v}\right) \tilde{v}-\mathscr{D}_{x} \tilde{p}=-\mathrm{i} \tilde{w} \tilde{u},} \\
-\left(\overline{\mathscr{L}}_{y} \bar{u}\right) \tilde{u}+\left[\mathscr{L}^{\dagger}-\left(\mathscr{P}_{y} \bar{v}\right)\right] \tilde{v}-\overline{\mathscr{L}}_{y} \tilde{p}=-\mathrm{i} \tilde{\omega} \tilde{v}, \\
\mathscr{L}^{\dagger} \tilde{u}+\mathrm{i} \beta \tilde{p}=-\mathrm{i} \tilde{w} \tilde{u},
\end{gathered}
$$

where $\mathscr{L}^{\dagger}=(1 / \operatorname{Re})\left(\bar{Q}_{x}^{2}+\mathscr{P}_{y}^{2}-\beta^{2}\right)+\bar{u} \bar{L}_{x}+\bar{v} \bar{L}_{y}$, and $\tilde{\omega}$ is the adjoint eigenvalue. In this context, the adjoint eigenmodes are defined as

$$
\tilde{\boldsymbol{q}}^{+}(x, y, z, t)=\tilde{\boldsymbol{q}}(x, y) \exp [\mathrm{i}(\beta z+\tilde{\omega} t)] .
$$

A solvability condition for the adjoint problem is obtained by enforcing that the bracketed terms in (3.7) vanish:

$$
[\langle\hat{\boldsymbol{v}}, \hat{\boldsymbol{v}}\rangle]_{0}^{t}+[\boldsymbol{J}(\check{\boldsymbol{q}}, \hat{\boldsymbol{q}})]_{\partial \Omega}=0 .
$$

The second term in (3.13) imposes the conservation of the bi-linear concomitant (Hill 1995; Dobrinsky \& Collis 2000) in the domain, and determines the boundary conditions for the adjoint problem on account of the boundary conditions for the direct problem.

Taking into account the intrinsic nature of the global mode of LSBs (Theofilis et al. 2000 ), Dirichlet boundary conditions are imposed on the disturbance components at inflow for the direct problem. Linear extrapolation is imposed at the outflow, as 
the physical behaviour of the disturbances is not known a priori. Consistently with the solvability condition, the inflow and outflow boundary conditions are inverted in the adjoint problem, i.e. Dirichlet homogeneous boundary conditions are used at the outflow and linear extrapolation at inflow. The velocity components of both the direct and adjoint eigenfunctions are imposed to vanish at the wall due to the no-slip condition. At the far field, the velocity and pressure disturbances are also forced to vanish. This last assumption is made on account of the exponential decay of the global modes along the wall-normal direction, and permits accurate recovery of this part of the eigenspectrum, although it is not necessarily adequate for the TS waves, and certainly is not justified for the continuous part of the spectrum (neither of which are of interest here). A compatibility condition is derived from the Navier-Stokes equations for $\partial \hat{p} / \partial y$ and is imposed for the pressure perturbation at the wall.

The direct and adjoint eigenvalue problems can be written in the form

$$
\begin{aligned}
\mathscr{J} \hat{\boldsymbol{q}}_{l} & =-\mathrm{i} \omega \omega_{l} g \hat{\boldsymbol{q}}_{l} \quad \text { and } \\
\mathscr{N} & \hat{\boldsymbol{q}}_{m}=-\mathrm{i} \tilde{\omega}_{m} \beta \hat{\boldsymbol{q}}_{m},
\end{aligned}
$$

where $\left(\omega_{l}, \hat{q}_{l}\right)$ and $\left(\hat{\sigma}_{m}, \tilde{\boldsymbol{q}}_{m}\right)$ are the direct and adjoint eigenpairs, respectively. Substituting (3.14) and (3.15) into (3.7), and taking into account the set of boundary conditions presented above, the solvability condition (3.13) yields the bi-orthogonality condition

$$
0=\left(-\mathbf{i} \omega_{l}-\left(-\mathbf{i} \tilde{\omega}_{m}\right)^{H}\right)\left\langle\tilde{\boldsymbol{q}}_{l},\left\langle s \hat{\boldsymbol{q}}_{m}\right\rangle .\right.
$$

Because of the inner product and Ansatz chosen in the development of the equations, $\omega_{r}=-\tilde{\omega}_{r}$ and $\omega_{i}=\tilde{\omega}_{i}$. The bi-orthogonality condition (3.16) imposes that the inner product of the adjoint and direct eigenvectors be equal to zero if $l \neq m$, i.e. when they do not correspond to the same eigenmode. In the case $l=m$, the term in parenthesis vanishes and the inner product is non-zero. It can be shown (Salwen \& Grosch 1981; Hill 1995) that if the eigenspectrum is complete, an arbitrary disturbance can be expressed as a linear combination of the direct eigenfunctions. The contribution of the disturbance to a discrete eigenmode $l$ is obtained as the projection of the disturbance on the adjoint eigenfunction $\tilde{q}_{l}$ using the condition (3.16), with an adequate normalization. Accordingly, the adjoint eigenfunctions describe the spatial regions of higher receptivity of the eigenmode to initial disturbances and external forcing. thus permitting the derivation of theoretically-founded active flow control strategies (Theofilis 2009). In the present context, solution of the adjoint eigenvalue problem alongside the concept of structural sensitivity, introduced in $\$ 3.3$, will aid in determining the circumstances under which the topologies exerted by the global mode can be realizable.

\subsection{Numerical methods}

The direct and adjoint eigenvalue problems constitute two generalized eigenvalue problems that are qualitatively identical, and in consequence the same numerical method is used for their independent solution. The two-dimensional partial-derivativebased system of equations (3.2)-(3.5) or (3.8)-(3.11) were discretized using ChebyshevGauss-Lobatto points in both of the $x$ and $y$ spatial directions. An algebraic mapping was used in the wall-normal direction in order to cluster points in the vicinity of the wall. After discretization, the systems of equations can be recast in the form

$$
\boldsymbol{A} X=\omega \boldsymbol{B} X
$$




$\begin{array}{lclc}\text { Problem } & \text { Domain size } & \text { Resolution } & \omega_{i} \times 10^{3} \\ \text { Direct } & (180,350) \times(0,35) & 340 \times 90 & -4.93855 \\ \text { Direct } & (152,370) \times(0,40) & 340 \times 90 & -4.93828 \\ \text { Direct } & (152,390) \times(0,40) & 340 \times 128 & -4.93845 \\ \text { Adjoint } & (152,370) \times(0,40) & 340 \times 90 & -4.93606\end{array}$

TABLE 2. Results of the convergence test for different resolutions and domain sizes.
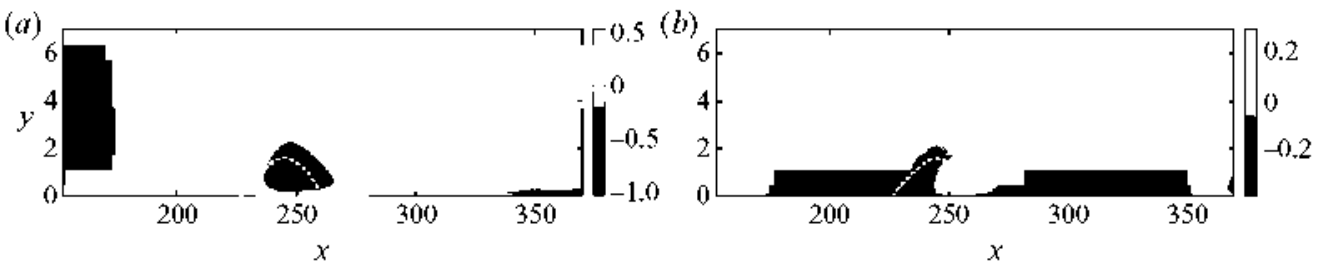

FIGURE 2. Streamwise $\hat{u}(a)$ and spanwise $\hat{w}(b)$ velocity components of the direct eigenfunction corresponding to the leading stationary eigenvalue for the case $\bar{\delta}_{\max }=4$, attained for $\beta=0.3$.

where the matrix $\boldsymbol{A}$ corresponds to either of the discretized operators $\mathscr{A}$ or $\mathscr{A}^{\dagger}$, and $B$ corresponds to $-\mathrm{i} \mathscr{B}$. The Amoldi algorithm with zero shift is used to recover a window of the complete eigenspectrum, whereby instead of (3.17), the problem solved is

$$
\overline{\boldsymbol{A}} X=\mu X, \quad \text { where } \quad \overline{\boldsymbol{A}}=\boldsymbol{A}^{-1} \boldsymbol{B}, \quad \mu=\frac{1}{\omega} .
$$

The Arnoldi iteration generates a Krylov subspace of dimension $m$, and a Hessenberg matrix with dimension $m \times m$, whose eigenvalues, obtained using the QZ algorithm, are approximations to those of the original eigenvalue problem. Taking advantage of the near-diagonal structure of the matrix $B$, only the left-hand-side matrix $\boldsymbol{A}$ is formed and stored. Dense linear algebra operations are used; in this context the memory requirements are well beyond the possibilities of a serial solution, requiring storage of and operating with matrices of $O\left(10^{5}\right)$ leading dimension. A parallel implementation of the algorithm is employed to make use of massively distributed memory architectures (Rodriguez \& Theofilis 2009). The largest resolution employed in the present analyses was $340 \times 128$ collocation points with Krylov subspace dimension $m=200$, requiring upwards of 460 Gbytes of memory and taking nearly four hours of wall-clock time on JUGENE (www.fz-juelich.de/jsc/jugene) for the solution of the eigenvalue problem at one value of the parameter $\beta$. Note that convergence of the eigenvalue is attained using a lower resolution, as shown in table 2 .

\subsection{Convergence studies and sensitivity of the eigenvalues}

Several convergence tests have been performed, and the results for the representative case $\bar{\delta}_{\max }=4$ and $\beta=0.3$ are summarized in table 2 . Using $340 \times 90$ collocation points in the baseline case, in which the full domain $L_{x} \times L_{y}=(152,370) \times(0,40)$ has been considered, the eigenvalue of the global mode - subject of the topological analysis that follows - is converged to within a relative error of $10^{-3}$ (absolute error of $10^{-6}$ ). The direct and adjoint eigenfunctions are shown respectively in figures 2 and 3. It should be noted that for two-dimensional basic flows with $\bar{w} \neq 0$ the change of variable $q=(u, v, w, p)^{T} \rightarrow(u, v, \mathrm{i} w, p)^{T}$ transforms the complex eigenvalue problems (3.2)(3.5) and (3.8)-(3.11) to real arithmetic (Theofilis 2003). With this transformation, 

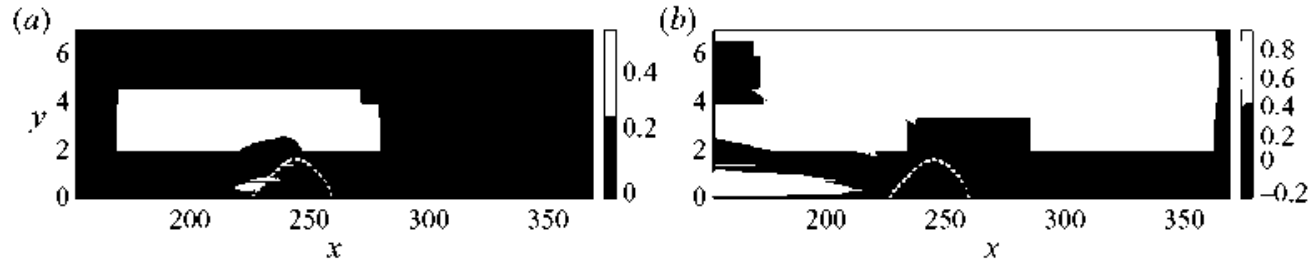

FIGURE 3. Streamwise $\tilde{u}(a)$ and spanwise $\tilde{w}(b)$ velocity components of the adjoint eigenfunction corresponding to the leading stationary eigenvalue for the case $\bar{\delta}_{\text {max }}=4$, attained for $\beta=0.3$.

the eigenvectors associated with real (stationary) eigenvalues, like the eigenmode considered here, are also real and then the spanwise velocity component $\hat{w}$ (or $\hat{w}$ ) is orthogonal to $\hat{u}$ and $\hat{v}$ (respectively $\tilde{u}$ and $\hat{v}$ ). In agreement with boundarylayer theory, the wall-normal velocity components are an order-of-magnitude smaller than the horizontal ones, and are not shown in the figures. As shown in Theofilis et al. (2000), the spatial structure of the direct eigenfunctions is centred inside the recirculation region and extends downstream, while the new result here concerns the adjoint eigenfunctions, which extend upstream. Furthermore, the maximum amplitude in the direct eigenfunctions is attained in the streamwise velocity component $\hat{u}$, while that in the adjoint eigenvector is attained in its spanwise component $\tilde{u}$.

The concept of sensitivity of the eigenvalues is employed here in order to determine the drift of the leading eigenvalue due to the combination of domain size and boundary conditions. It can be shown (Schmid \& Henningson 2001; Luchini 2003; Chomaz 2005; Giannetti \& Luchini 2007) that the eigenvalue drift $\delta \omega_{i}$ due to a spatially localized perturbation $\delta \boldsymbol{A}_{0}$ of the linear operator $\boldsymbol{A}$, comprising variations of the basic flow and boundary conditions, has an upper bound defined by

$$
\delta \omega_{l} \leqslant\left\|\delta \boldsymbol{A}_{\hat{\theta}}\right\| \boldsymbol{s}_{l}(x, y), \quad \text { where } \quad \boldsymbol{s}_{l}(x, y)=\frac{\left\|\tilde{\boldsymbol{q}}_{l}\right\| \cdot\left\|\hat{\boldsymbol{q}}_{l}\right\|}{\left\langle\tilde{\boldsymbol{q}}_{l}, \boldsymbol{B} \hat{\boldsymbol{q}}_{l}\right\rangle} .
$$

The function $s_{f}(x, y)$, different for each eigenmode, determines the spatial locations where perturbations of either the basic flow or the boundary conditions have the largest effect on the resulting eigenvalue. The maxima of $s_{l}$ are the locations at which a higher coupling between the respective direct and adjoint eigenfunctions exists. The structure of the direct-adjoint coupling function $s$ for this eigenmode, shown in figure $(4 a)$, is highly localized inside the recirculation region.

Giannetti \& Luchini (2007) noted that the sensitivity of the eigenvalue to the size of the computational domain, or equivalently to the boundary conditions, can be predicted by considering the spatial structure of $s$. In particular, the eigenvalue drift (3.19) caused by the domain truncation and choice of boundary conditions will be, at most, of the same order of magnitude as $s$. Contours of $\log _{10}(s)$ are shown in figure ( $4 b$ ) along with the boundaries of the smaller domain considered for the convergence test (table 2). The maximum value of $s$ at the boundaries of the smaller domain is $O\left(10^{-5}\right)$, while the difference on the computed eigenvalues is $O\left(10^{-7}\right)$. Consequently, the eigenvalue is recovered accurately both within the baseline and the smaller domain $L_{x} \times L_{y}=(180,350) \times(0,35)$ shown in figure 4 .

\subsection{BiGlobal linear instability results and their structural sensitivity}

Stability analyses were performed for the range of spanwise wavenumbers $\beta \in[0,1.5]$, corresponding to periodicity lengths varying from the two-dimensional case $L_{\bar{z}} \rightarrow \infty$ 

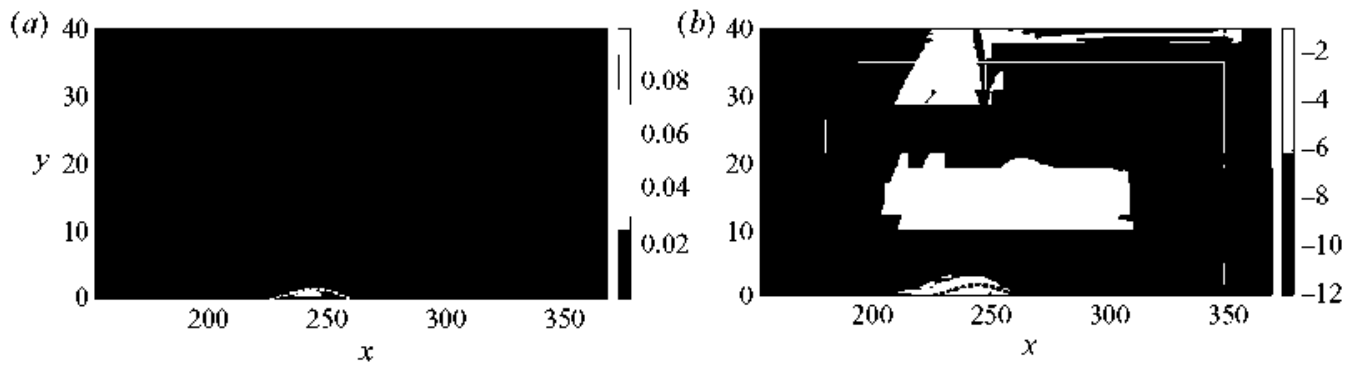

FIgure 4. Contours of the direct-adjoint coupling function $s(a)$ and $\log _{10}(s)$ (b) corresponding to the leading stationary eigenvalue for the case $\bar{\delta}_{\max }=4$, attained for $\beta=0.3$. The white lines show the boundaries of the smaller domain considered in the convergence test.

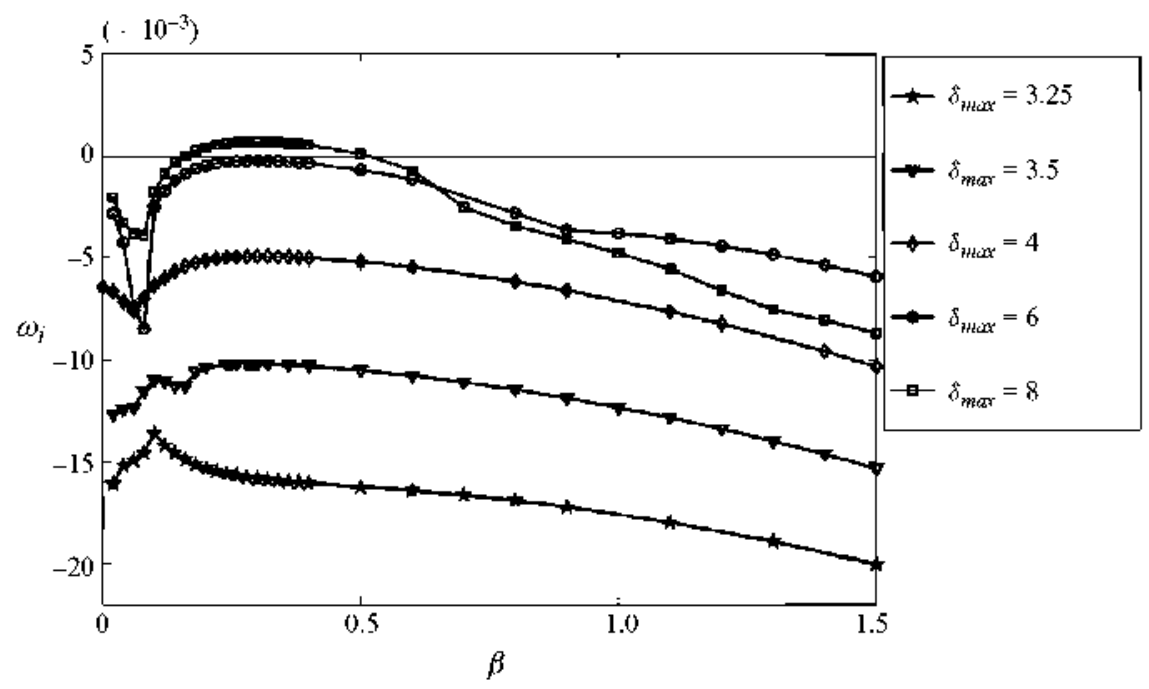

FIGURE 5. Dependence of the growth rate of the leading stationary global mode with the spanwise wavenumber $\beta$ for different laminar separation bubbles.

to $L_{3} \approx 4$, an order of magnitude smaller than the streamwise extension of the LSBs considered. The dependence of the growth rate $\omega_{i}$ of the stationary global mode with the spanwise wavenumber $\beta$ for some of the LSBs defined in table 1 is shown in figure 5. In the case of the weakest bubble, $\bar{\delta}_{\max }=3.25$, the least damped mode corresponds to $\beta=0.15$. As the separation bubble becomes stronger, the spanwise wavenumber $\beta \approx 0.3$ becomes dominant. The dominant eigenmode is increasingly less damped with stronger separation bubbles, and eventually it becomes unstable for $\bar{\delta}_{\max } \approx 7$, concurrently with the appearance of a second dominant wavenumber $\beta \approx 1$.

As can be seen in table 1, the appearance of a self-excited global mode in the present context occurs at a level of recirculation of $u_{r e r} \approx 6 \%-7 \%$, consistently with earlier BiGlobal results and in contrast with the substantially higher values delivered by absolute/convective analysis (Hammond \& Redekopp 1998). The structure of the eigenfunctions corresponding to the leading eigenmode for the different basic flows is qualitatively identical to the one shown in figures 2 and 3 , and exhibit the same characteristics of those known from earlier work on the flat plate (Theofilis et al. 2000) and from instability analysis of related flows (Theofilis et al. 2002; Gallaire 
et al. 2007; Robinet 2007; Abdessemed et al. 2009; Kitsios et al. 2009; Marquet et al. 2009).

The leading stationary global mode has been encountered to be stable for all the range of wavenumber $\beta$ for LSB models with $\bar{\delta}_{\max } \leqslant 7$, and temporally amplified for a range of finite spanwise wavenumbers if $\bar{\delta}_{\max } \geqslant 7$. The maximum receptivity to external perturbations of the flow, characterized by the adjoint eigenfunctions, is located at a spatial region near the basic flow separation line, extending upstream beyond the inflow boundary, as can be seen in figure 3 . The concept of structural sensitivity (Giannetti \& Luchini 2007), introduced as a physical interpretation of the eigenvalue sensitivity, states that the mechanism responsible for a self-excited eigenmode acts in the spatial regions defined by $s$. Consequently, perturbing the flow in the regions of highest sensitivity produces the largest eigenvalue drift. Provided that the growth/damping rates are $O\left(10^{-3}\right)$, the results on $\log _{10}(s)$ in figure $(4 b)$ show that small perturbations of the basic flow in the region of highest sensitivity, would suffice to displace the eigenvalue up to the point of changing its sign and thus alter its instability behaviour from stable to unstable. In this context, the topological changes exerted by the stationary global mode, as described in $\$ 5.3$, can exist either as a result of a self-excited (unstable) or as a continuously excited (stable) disturbance. Incidentally, the same information on structural sensitivity can also be used in order to devise efficient passive flow control strategies, as shown in the case of the cylinder by Giannetti \& Luchini (2007).

\section{Direct numerical simulation}

\subsection{Numerical method}

The results of the BiGlobal instability analysis have been verified by linear and nonlinear three-dimensional DNS monitoring the linear development of the disturbance velocity components associated with the stationary leading global mode. The DNS code is a modification of that of Lundbladh et al. (1994) and has also been used by Theofilis et al. (2003) in the verification of the polynomial modes of swept Hiemenz flow. It considers a velocity-vorticity formulation of the incompressible continuity and Navier-Stokes equations. Chebyshev polynomials are used for the wall-normal direction; here a mapping and domain extension identical with those used in the stability analysis have been implemented in the DNS code. A CrankNicholson scheme is used for the time advancement of the viscous terms while a third-order Runge-Kutta scheme is used for the nonlinear terms.

The two horizontal directions are considered homogeneous and treated as periodic with a Fourier discretization. However, this is only consistent for the spanwise direction $z$. In order to obtain periodicity in the streamwise direction, a fringeregion technique (Spalart 1988) is used, consisting of adding a linear damping term to the governing equation

$$
\frac{\partial \boldsymbol{v}}{\partial t}+\mathscr{f}(\overline{\boldsymbol{v}}, \boldsymbol{v})=f(x)(\boldsymbol{V}-\boldsymbol{v}),
$$

where $v=(u, v, w)^{T}$ is the disturbance velocity, $\mathscr{S}$ is the spatial operator, $\bar{v}$ is the frozen basic flow, and $\boldsymbol{f}(x)$ is the fringe function. The right-hand-side term in (4.1) forces the solution $v$ towards $\boldsymbol{V}$. The function $\boldsymbol{V}$ can be arbitrarily chosen in order to introduce inflow boundary condition or forcing terms. In line with the homogeneous Dirichlet boundary conditions imposed in the BiGlobal eigenvalue problem, for the present computations the value of $V$ is set to be identically zero. The fringe function 
$f(x)$ is chosen to minimize the influence of the fringe region on the physically relevant part of the domain:

$$
f(x)=\hat{f} S(\xi), \text { where } \quad \xi=\frac{x-x_{\text {start }}}{\Delta} .
$$

The parameter $\hat{f}$ defines the magnitude of the fringe function, while the parameters $x_{\text {star }}$ and $\Delta$ define the shape of the function. The function $S(\xi)$ used here is identical with that used in the definition of the basic flow displacement thickness distribution (2.10).

\subsection{Initial and botndary conditions}

Direct numerical simulations were performed in the three-dimensional computational boxes $L_{x} \times L_{y} \times L_{z}=(152,390) \times(0,40) \times(0,2 \pi n / \beta)$, with $n=1, n=2$ and $n=5$ respectively, containing $n$ complete spanwise wavelengths of the dominant eigenmode. A resolution of $(N x \times N y \times N z)=(360 \times 128 \times 32)$ spectral points has been used in the streamwise, wall-normal and spanwise spatial directions, respectively. The fringe region is defined within $x \in[370,390]$ and is resolved by 34 Fourier collocation nodes. An additional basic flow and linear instability analysis with a longer domain were also computed; as seen in the third row of results in table 2 , the effect of this modification on the damping rate is negligible. For consistency with the BiGlobal instability analysis, the same basic flows $\bar{v}$ delivered by the boundary-layer approximation are used here too, and are kept frozen in the computation. The DNS was initialized by the scaled amplitude functions of the disturbance velocity components corresponding to the leading stationary global eigenmode at a given small amplitude $\varepsilon=10^{-2}$. Consistently with the BiGlobal instability analyses, vanishing of the disturbance velocity was imposed at the wall and at the far field, while adequate Neumann boundary conditions were derived for the disturbance vorticity components.

Two different methods have been used in order to extract the growth/damping rates from the DNS. The first method considers a measure of the kinetic energy at a given wavenumber $k_{z}$ in the spanwise Fourier expansion, defined as

$$
E\left(k_{z}, t\right)=\frac{1}{L_{x}^{\prime}} \int_{0}^{L_{y}}\left[\int_{0}^{L_{j}} \frac{1}{2} \boldsymbol{v}^{2}\right] \mathrm{d} y .
$$

where the streamwise extension $L_{x}^{\prime}$ is selected such that the influence of the fringe region be negligible. The dominant growth rate for the specific wavenumber $k_{i}=\beta$ is calculated from the time signal of $E\left(k_{z}, t\right)$ : the time derivative is computed using (first-order) two-point backward finite differences at each time step, resulting in a near-constant approximation of the amplification/damping rate, $2 \omega_{i}$; finally, an average of the latter quantity over the simulation time is calculated. The selection of the length $L_{x}^{\prime}$ for the energy integral being arbitrary, a second method has also been used, that overcomes this uncertainty. The evolution of the maximum magnitude of the spanwise disturbance velocity component in the entire domain, $\max \{\|\hat{w}\|\}$, is also monitored and the growth/damping rate is extracted in an analogous manner from its averaged time derivative.

\subsection{Validations; linear and nonlinear DNS runs}

The basic flow corresponding to $\bar{\delta}_{m a x}=4$ along with its leading stationary global mode, which is stable at the spanwise wavenumber $\beta=0.3$, is considered for the validation of the results of the instability analysis. Note that this case is the same considered for the convergence test shown in $\$ 3$. The results are summarized in table 3; 


$\begin{array}{lccc}\text { Case } & n=\beta L_{z} / 2 \pi & \omega_{i, D N S, \hat{E}} \times 10^{3} & \omega_{i, D N S, \hat{x}} \times 10^{3} \\ \text { Linear } & 1 & -4.762 & -4.965 \\ \text { Linear } & 2 & -5.029 & -4.910 \\ \text { Linear } & 5 & -4.991 & -4.898 \\ \text { Nonlinear } & 1 & -4.447 & -4.938\end{array}$

TABLE 3. Temporal growth/damping rates, relative to the leading stationary global mode for the case $\bar{\delta}_{m a x}=4$ and $\beta=0.3$, extracted from three-dimensional direct numerical simulations.

shown are four different simulations, three of which were performed neglecting the nonlinear terms and considering different spanwise extensions, while in the fourth simulation the nonlinear terms were re-instated. A noticeable dispersion is observed on the recovered damping rate values when using the kinetic energy approach, while all results are consistent with the value predicted by the instability analysis. This is attributed to two effects, firstly the arbitrariness in the choice of $L_{x}^{\prime}$ and the potential for the fringe region to contaminate the results and secondly the fixed resolution in the spanwise spatial direction. The agreement between eigenvalue problem and DNS results is improved substantially when the peak spanwise velocity is considered: the largest absolute error between the results of table 3 with the linear theory result, $\omega_{i, L S T}=-4.93845 \times 10^{-3}$, is $\approx 4 \cdot 10^{-5}$, while the maximum relative error is $0.8 \%$.

\section{Structural changes exerted by the stationary global mode}

\subsection{Topological considerations}

The organized structures in a flow field can be characterized through the identification of the physical locations where the velocity vanishes (i.e. critical points), the behaviour of the streamlines in the vicinity of these points, and the manner in which the critical points are connected by the dividing streamlines. An exhaustive description of the theory of critical points for compressible three-dimensional unsteady laminar or turbulent flows can be found in several sources (Dallmann 1982; Homung \& Perry 1984; Perry \& Hornung 1984; Perry \& Chong 1987; Chong. Perry \& Cantwell 1990; Dallmann et al. 1997); for completeness, the main aspects concerning incompressible flows are summarized next.

A general three-dimensional flow field is expressed by the vector field $\boldsymbol{v}=(u, v, w)$ defined on the coordinate system $x=(x, y, z)$. Critical points are defined as the spatial locations where the three components of the velocity are equal to zero and the slope of the streamlines is undetermined. The flow field then can be locally expanded around the critical points using Taylor series. Considering only the linear terms in the expansion, the local behaviour of the streamlines is determined by the Jacobian matrix $J$ :

$$
\left(\begin{array}{l}
\dot{x} \\
\dot{y} \\
\dot{z}
\end{array}\right)=\left(\begin{array}{lll}
j_{11} & j_{12} & j_{13} \\
j_{21} & j_{22} & j_{23} \\
j_{31} & j_{32} & j_{33}
\end{array}\right)\left(\begin{array}{l}
x \\
y \\
z
\end{array}\right) .
$$

where $j_{i j}$ are real constants, and the critical point has been set as the origin of the coordinate system. In the case of free-slip points, i.e. critical points in the absence of a solid wall, $j_{i j}$ are the elements of the rate-of-deformation tensor evaluated at the origin, $(x, y, z)=(0,0,0)$. A second type of critical points (denominated no-slip points) exists, in which the slope of the streamlines is undetermined on a solid wall. 
Equation (5.1) can still be used for characterizing the properties of no-slip critical points, introducing $\left({ }^{\circ}\right)=\mathrm{d}() \mathrm{d} \tau$. The transformed time variable $\tau$ is defined by $\mathrm{d} \tau=y \mathrm{~d} t$ where $y$ is the wall normal coordinate. The elements of the Jacobian matrix no longer correspond to the rate-of-stress tensor, but second derivatives of its elements in $y$ (e.g. $\left.j_{11}=\partial^{2} u / \partial x \partial y+\partial^{2} v / \partial x^{2}\right)$. The limit $y \rightarrow 0$ defines the surface streamlines or lines of surface shear stress.

In the most general case there are three planes containing solution trajectories originating at the critical point. These planes are defined as the eigenvector planes of the Jacobian matrix. Let $\lambda$ be the eigenvalues of the Jacobian, satisfying the characteristic equation

$$
\lambda^{3}+P \lambda^{2}+Q \lambda+R=0,
$$

where $P, Q$ and $R$ are the scalar invariants of the Jacobian matrix,

$$
\begin{aligned}
& P=-\left(j_{11}+j_{22}+j_{33}\right), \\
& Q=\left|\begin{array}{ll}
j_{11} & j_{12} \\
j_{21} & j_{22}
\end{array}\right|+\left|\begin{array}{ll}
j_{11} & j_{13} \\
j_{31} & j_{33}
\end{array}\right|+\left|\begin{array}{ll}
j_{22} & j_{23} \\
j_{32} & j_{33}
\end{array}\right|
\end{aligned}
$$

and

$$
R=-\left|\begin{array}{lll}
j_{11} & j_{12} & j_{13} \\
j_{21} & j_{22} & j_{23} \\
j_{31} & j_{32} & j_{33}
\end{array}\right| .
$$

The invariant $P$ is the divergence of the velocity field, which is identically equal to zero due to incompressibility. The cubic characteristic equation (5.2) can have (i) three different real roots, (ii) all real roots with at least two of them equal and (iii) one real- and a pair of complex roots. Real and complex solutions are separated in the $Q-R$ space by the curve

$$
S_{1}=27 R^{2}+4 Q^{3}=0 .
$$

Complex eigenvalues are associated with $S_{1}>0$ and real eigenvalues with $S_{1}<0$. In the most general case the eigenvectors are non-orthogonal. The Jacobian matrix $J$ can be transformed to another matrix $J^{\prime}$ with the same eigenvalues through the transformation

$$
\boldsymbol{x}^{\prime}=\mathbf{T} \cdot \boldsymbol{x}
$$

in which the eigenvectors are orthogonal and where the new coordinates $\boldsymbol{x}^{\prime}$ are measured in the direction of the eigenvectors. The transformed Jacobian

$$
\dot{x}^{\prime}=\boldsymbol{J}^{\prime} \cdot \boldsymbol{x}^{\prime}
$$

is then said to be in canonical form. The study of the local trajectories of the streamlines is more conveniently done in the canonical form. The solution trajectories in the direction of an eigenvector associated with a real positive (negative) eigenvalue are expelled from (attracted to) the critical point. When a pair of complex eigenvalues exists, the streamlines describe spiral trajectories in the plane defined by the two eigendirections. The real parts of the eigenvalues determine whether the streamlines are attracted or repelled by the critical point. Once the location and classification of the critical points is known, the streamlines connecting the critical points completes the description of the flow field.

The classification of the critical points in a three-dimensional flow field can be done in two ways. The first possibility is to consider the type of critical point arising from 


$\begin{array}{llccccc} & x-y & x-z & y-z & R & Q & S_{1} \\ \text { B1 } & \text { S } & - & - & 0 & <0 & <0 \\ \text { B2 } & \text { S } & - & - & 0 & <0 & <0 \\ \text { B3 } & \text { C } & - & - & 0 & >0 & >0 \\ \text { L1/H1 } & \text { S } & \text { S } & \text { N(u) } & >0 & <0 & <0 \\ \text { L2 } & \text { S } & \text { N(s) } & \text { S } & <0 & <0 & <0 \\ \text { L3 } & \text { S } & \text { N(u) } & \text { S } & >0 & <0 & <0 \\ \text { L4/H4 } & \text { S } & \text { S } & \text { N(s) } & <0 & <0 & <0 \\ \text { L5 } & \text { F(u) } & \text { S } & \text { S } & >0 & - & >0 \\ \text { L6/H6 } & \text { F(s) } & \text { S } & \text { S } & <0 & - & >0 \\ \text { H35d } & \text { S } & \text { NF } & \text { S } & >0 & <0 & =0 \\ \text { H35 } & \text { S } & \text { F(u) } & \text { S } & >0 & <0 & >0\end{array}$

TABLE 4. Classification of critical points according to the projection on the coordinate planes and the location on the $Q-R$ plane. $\mathrm{S}$ : saddle; $\mathrm{C}$ : centre; $\mathrm{N}$ : node; $\mathrm{F}$ : focus; $\mathrm{NF}$ : Node focus; (s): stable; (u): unstable. Points named with 'B', ' $\mathrm{L}$ ' and ' $\mathrm{H}$ ' correspond to the basic flow, lower disturbance amplitude and higher disturbance amplitude topologies, respectively.

three cross-sections or projections onto the coordinate planes: two real eigenvalues with the same sign define a node, two real eigenvalues with opposite sign define a saddle point, and a pair of complex eigenvalues defines a focus - if the real part is different from zero - or a centre. The three-dimensional critical point is then defined in terms of 'triads' (e.g. node-saddle-saddle). Erroneous classifications are possible using this methodology, if the sectional planes are not adequately selected. The second method of identification of critical points uses the values of the invariants $R$ and $Q$. and the relative location of the curve $S_{1}$ to classify the triads. This method avoids the possibility of errors in the classification of the critical points, but the physical insight of the critical point and connecting streamlines is less apparent. In accordance, both methodologies are employed in this paper. The classification of the critical points appearing in the paper is summarized in table 4 .

The sectional streamlines, or streamlines on cross-sections, are also useful in the identification of swirling motions. Critical points appear in the cross-sections which are not critical points of the three-dimensional flow as the velocity normal to the plane does not vanish. However, they provide information on how the spiralling streamlines behave.

The structures of two different flow fields are said to be topologically equivalent if the classification and connections of the critical points are identical, even though the velocity magnitudes or critical point locations are different. Nevertheless, bifurcations in the topology description can appear under small changes in the flow field. 'Local' bifurcations are those related to (i) the appearance or disappearance of critical points, or (ii) changes in the eigenvalue classification of the local Jacobian. 'Global' bifurcations are related with changes in the streamline connections between critical points. The borderline topology between two different topology descriptions is said to be structurally unstable (Guckenheimer \& Holmes 1983). This can be due to either critical points where at least one eigenvalue is $\lambda=0$ ('local degeneracy') or saddle-to-saddle connections ('global degeneracy'). In any case, a random small perturbation on the structurally unstable flow field will alter the description of the topology. 

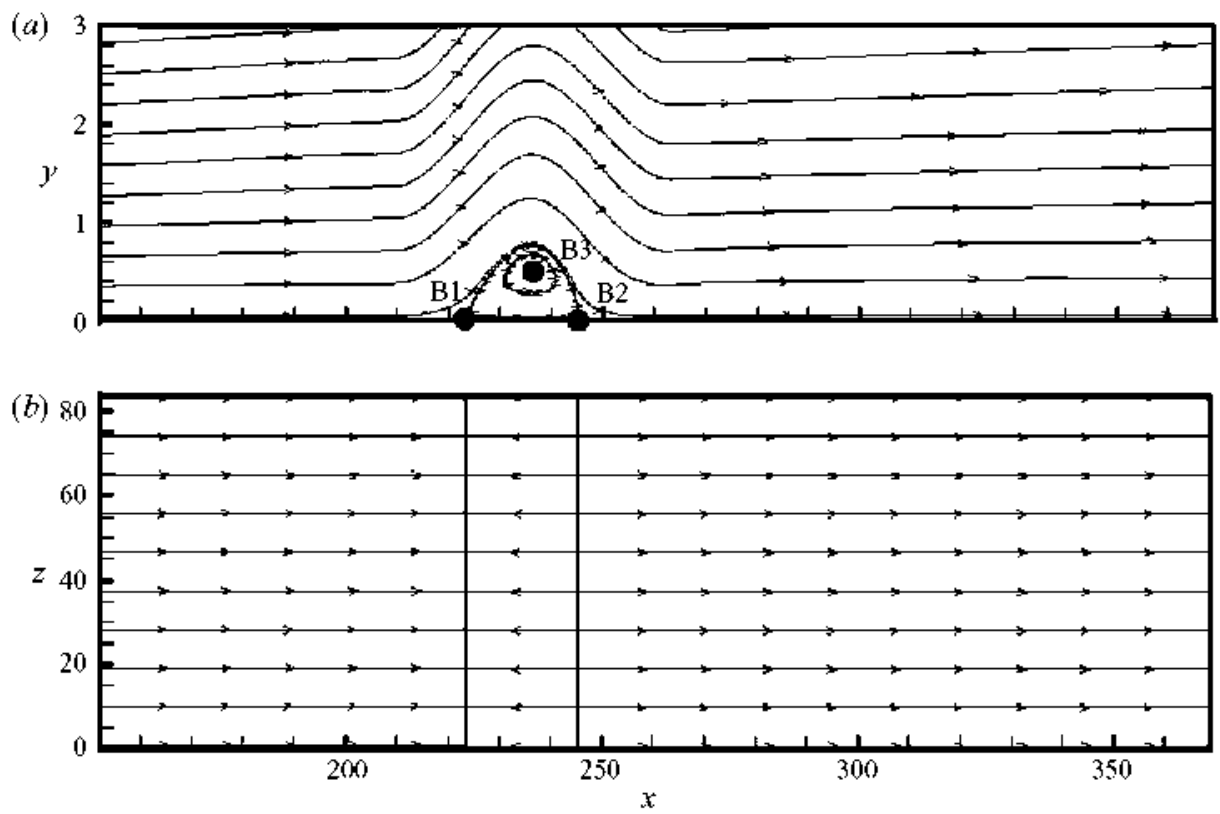

FIGURE 6. Streamlines of the basic flow for $\bar{\delta}_{\max }=3.25$. (a) Plane $z=0$, (b) plane $y=0$.

\subsection{Topology of the two- and three-dimensional laminar separation bubble flows: general remarks}

The streamlines corresponding to a representative basic flow are depicted in figure 6 . The case considered was $\bar{\delta}_{\max }=3.25$, but all the basic flows shown in table 1 are topologically equivalent, and the arguments presented in this section are valid for any LSB flow. The basic flow is two-dimensional and homogeneous in the spanwise direction: the velocity $\bar{w}$ and its derivatives with respect to $x$ and $y$, as well as all flow derivatives with respect to $z$ are equal to zero. Consequently the only non-zero terms in the Jacobian matrix are $j_{11}, j_{12}, j_{21}$ and $j_{22}$, while the invariant $R=0$. According to boundary-layer theory, in the vicinity of the wall $\partial / \partial x \ll \partial / \partial y$, and $v \ll u$. Separation and reattachment occur in two no-slip critical points (denominated B1 and B2 in table 4) on the vertical plane $O x y$, where $j_{12} \sim \partial^{2} u / \partial y^{2}>0$ and $j_{21} \sim \partial^{2} v / \partial x \partial y \approx 0$. At separation the term $j_{11}<0\left(j_{11}>0\right.$ at reattachment $)$ and continuity implies that $j_{11}=-j_{22}$. Therefore these critical points are classified as saddle points (two real eigenvalues with opposite sign, or $Q<0, S_{1}<0$ ). A third free-slip critical point is also present, where $j_{11}=j_{22}=0$, the terms $j_{12}$ and $j_{21}$ are finite valued and the invariant $Q>0$. The resulting pair of pure complex eigenvalues defines a centre (point B3), the streamlines following closed trajectories around it. The flow being homogeneous in the spanwise direction, the Jacobian matrix in all critical points contains the eigenvalue $\lambda=0$ associated with this direction. The locus of the critical points on different vertical planes gives rise to the separation and reattachment lines, and to a 'vortex' which is homogeneous in the third, out-of-plane spatial direction. The plane streamlines emanating from the separation saddle are necessarily connected to the reattachment saddle, engulfing the recirculation region.

This topology is structurally unstable and only possible in strictly two-dimensional flows; any small three-dimensional perturbation will give rise to new critical points and consequently to a new three-dimensional topology, regardless of the amplitude 
considered. The location of the new set of critical points is determined by the vanishing of the disturbance spanwise velocity component, and their classification is determined by the Jacobian of the perturbation.

For the sake of exposition of our argument, let us consider the Jacobian matrix $J$ of a three-dimensional flow field constructed by linearly superposing to the basic flow a three-dimensional perturbation with small amplitude $\varepsilon \ll 1$, evaluated at the recirculation centre. Evaluated at this critical point, the terms $\partial u / \partial x=\partial v / \partial y=0$, and then $\bar{j}_{11}=\bar{j}_{22}=0$. Moreover, $\partial u / \partial y>0$ and $\partial v / \partial x<0$. The total (i.e. basic flow plus linear perturbation) Jacobian takes the form

$$
J=\left(\begin{array}{ccc}
0 & \bar{j}_{12} & 0 \\
\bar{j}_{21} & 0 & 0 \\
0 & 0 & 0
\end{array}\right)+\varepsilon\left(\begin{array}{lll}
\hat{j}_{11} & \hat{j}_{12} & \hat{j}_{13} \\
\hat{j}_{21} & \hat{j}_{22} & \hat{j}_{23} \\
\hat{j}_{31} & \hat{j}_{32} & \hat{j}_{33}
\end{array}\right),
$$

where the elements $\bar{j}_{i j}$ correspond to the basic flow and $\hat{j}_{i j}$ correspond to the perturbation. The characteristic equation defining the eigenvalues of the disturbed critical point, is

$$
\lambda^{3}+\lambda \bar{j}_{12} \cdot \bar{j}_{21}+\varepsilon\left\{\bar{j}_{12} \cdot \bar{j}_{21} \cdot \hat{j}_{33}\right\}+\ldots=0 .
$$

For consistency with the linearization assumption, higher order terms are not retained. The equation considering only the order unity terms is the same as that for the basic flow and yields the same eigenvalues $\left(\lambda_{1,2}= \pm \mathbf{i} \sqrt{\bar{j}_{12} \bar{j}_{21}}\right.$ and $\left.\lambda_{3}=0\right)$ discussed earlier. The $O(\varepsilon)$ terms affect the eigenvalues $\lambda_{1}$ and $\lambda_{2}$ as linearly small corrections, necessary to verify the incompressibility condition; however, now $\lambda_{3}=\varepsilon \hat{j}_{33} \neq 0$, and as a consequence, $R \neq 0$. The classification of the critical point changes from the degenerate two-dimensional centre to a three-dimensional unstable (stretching) focus, when $\hat{j}_{33}>0$, or a stable (compressing) focus when $\hat{j}_{33}<0$. Similar developments can be derived for the other critical points present in the basic flow, namely the separation and reattachment points. The location of the critical points in the three-dimensional flow fields varies as the perturbation amplitude is increased and new bifurcations on the topology can appear. This is the case for the stationary global mode, discussed in next section.

\subsection{The stationary global mode}

Three-dimensional flow fields are reconstructed superposing to the basic flow, $\bar{v}$, the leading stationary three-dimensional global mode at linearly-small amplitudes. The basic flow corresponding to $\bar{\delta}_{\max }=3.25$ and its leading stationary mode are considered here. The choice of this case is made on account of the relatively simple wall-shear distribution of the basic flow, shown in figure 1 . As can also be seen in the same figure, basic LSB flows corresponding to higher values of $\bar{s}_{\max }$ have a wall-shear distribution which is increasingly more involved, comprising new inflection points. The existence of these inflection points has been seen to result in the appearance of additional critical points as the disturbance amplitude increases, and subsequently to additional topological bifurcations. However, all $\bar{\delta}_{\max }$ cases share at least two topologies: the lower-disturbance-amplitude reconstruction, corresponding to the onset of threedimensionality for infinitesimal disturbances and the higher-disturbance-amplitude reconstruction corresponding to the final topology studied herein. The topological bifurcations exerted by the global mode at higher $\bar{\delta}_{\max }$ values will not be discussed here, since the main features of the perturbed flow remain identical with those 

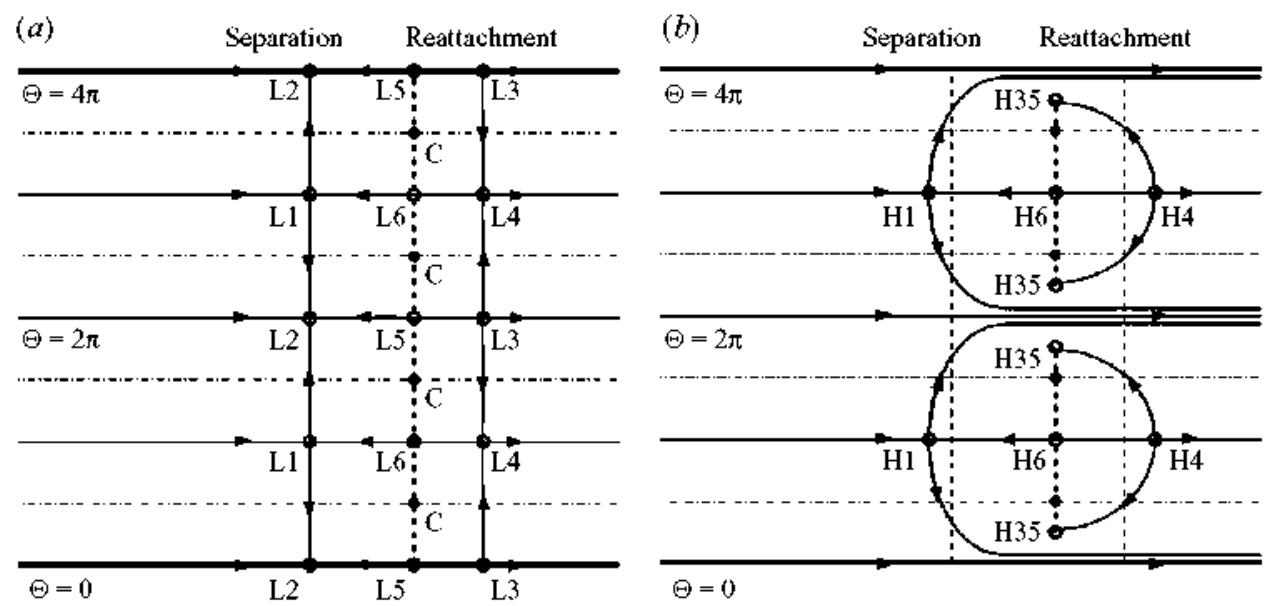

FIGURE 7. Surface critical points description for stationary the global mode with infinitesimal amplitude $(a)$ and with the higher amplitude $(b)$.

presented in what follows, although the bifurcations themselves are substantially more involved.

In order to construct the composite flow field, the eigenfunction is normalized to have the maximum streamwise velocity component equal to unity, and is then added linearly to the basic flow according to (3.1), with a given small amplitude $\varepsilon$. The three-dimensional domain considered for the reconstruction of the flow is $L_{x} \times L_{y} \times L_{z}=(152,370) \times(0,40) \times(0,4 \pi / \beta)$, thus containing two complete spanwise periods.

As mentioned earlier, the amplitude functions of the global mode recovered presently exhibit the same characteristics of those known from the earlier work (Theofilis et al. 2000). The streamwise velocity component $\hat{u}$ has a peak region that extends from the maximum wall-normal height of the bubble until the reattachment location in the basic flow. The maximum negative and positive streamwise disturbance velocities are attained for the phase planes $\Theta=0$ and $\pi$, respectively. A spanwise velocity component $\hat{u}$ with amplitude of the same order of magnitude as $\hat{u}$ is also present. The amplitude function changes its sign in some location between the separation and reattachment lines, and attains its minimum and maximum in counterphase (i.e. in $\Theta=\pi / 2,3 \pi / 2$ planes, respectively); the result is a fully three-dimensional eigenmode.

No-slip critical points appear at the planes $\Theta=0, \pi$, where the spanwise velocity vanishes. Referring to the schematic representation of figure 7 , on the $\Theta=0$ separation line $\hat{j}_{33}<0$ and a stable node (L1: $Q<0, R<0, S_{1}<0$ ) appears at the surface. The centre present in the basic flow is transformed into an unstable focus (L5: $\left.Q<0, R>0, S_{1}>0\right)$ : In the critical point $\hat{j}_{33}<0$, continuity forcing the other two terms in the diagonal to be positive. Conversely, in the reattachment line both $j_{11}$ and $j_{33}$ change sign, and an unstable node (L3: $Q<0, R>0, S_{1}<0$ ) appears. A similar discussion shows that on the plane $\Theta=\pi$ saddle points appear both at the separation (L2: $Q<0, R>0, S_{1}<0$ ) and the reattachment (L4: $Q<0, R<0, S_{1}<0$ ) lines, and that the centre is replaced by a stable focus (L6: $Q<0, R>0, S_{1}>0$ ).

It is worth mentioning that the flow topology described herein is true, independently of the actual magnitude of the (small-) amplitude parameter used to reconstruct the three-dimensional flow field. This is a consequence of the breakdown of the degenerate 


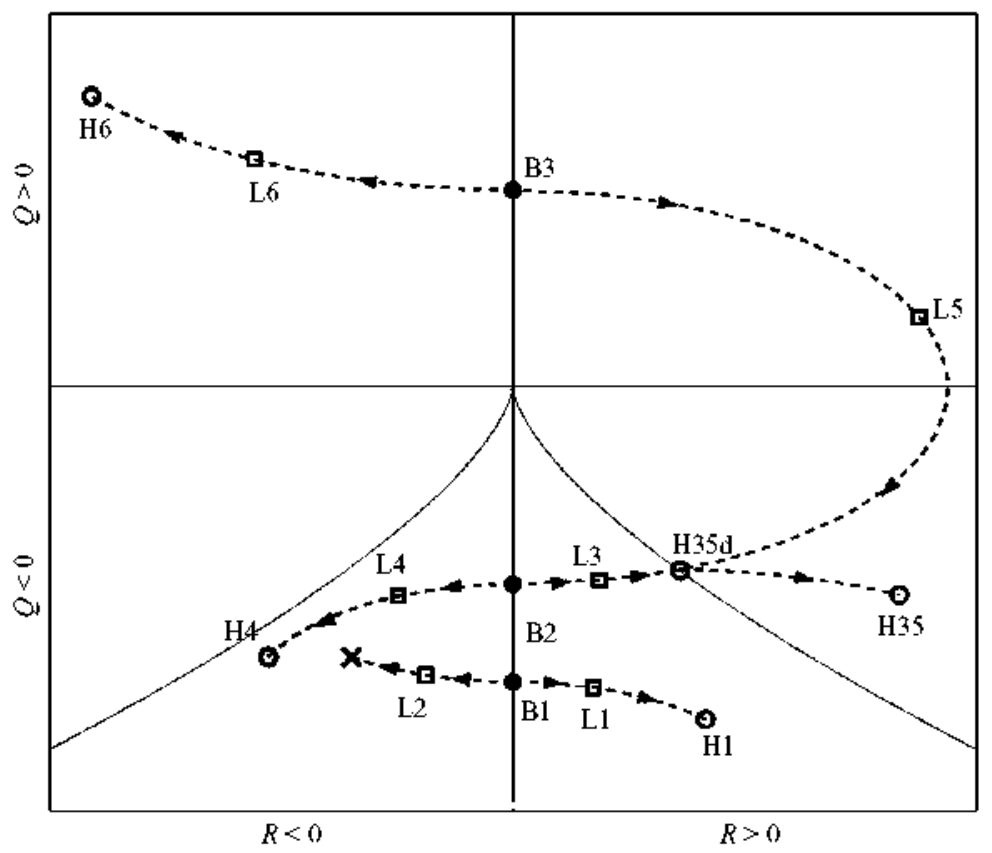

FIgure 8. Critical points location in the $Q-R$ plane. The arrows show qualitatively the evolution of the critical points with increasing amplitude $\varepsilon$.

two-dimensional structure of the basic flow, according to the discussion of $\$ 5.2$. The topology remains structurally equivalent if finite amplitudes are considered in the flow reconstruction, while the qualitative description of the disturbed flow in the latter case is more clearly visible. The deceleration of the basic flow $(\hat{u}<0)$ increases locally the streamwise extension of the reversed flow region. A fluid particle entering the domain sufficiently near the wall in the plane $\Theta=\pi$ is attracted into the stable focus describing a plane trajectory, and afterwards is expelled in the spanwise direction following a stretching spiral. When the maximum or minimum $\hat{w}$-values are reached (on the planes $\Theta=\pi / 2$ and $3 \pi / 2$ ), the spiral focus turns from attracting streamlines to repelling them, and the critical point on the sectional vertical plane is a centre. In the region $\pi / 2<\Theta<3 \pi / 2$ the streamlines are repelled by the compressing (unstable) focus, finally escaping the separation bubble and being convected downstream by the mean flow.

The streamline trajectories and the location of the critical points for a flow reconstruction using the global mode with an amplitude $\varepsilon=0.1$ is shown in the upper part of figure 9. The amplitude $\varepsilon$ used for the reconstruction allows a better visualization of the topological changes exerted by the linear mode while remaining topologically equivalent to a reconstruction with infinitesimal amplitude. In contrast to the two-dimensional basic flow in which a separatrix exists, isolating the trajectories inside the recirculation region and those entering the domain and escaping through the outflow, here no such closed trajectories exist on account of the presence of the global mode. The location and eigenvalues of the critical point is continuously modified by the disturbance amplitude, as illustrated in figure 8 where the loci of the critical points on the $Q-R$ plane are shown. The topology remains structurally equivalent to the one formerly described (points $L$ ) for a range of finite amplitudes. However, a new local bifurcation of the flow topology appears when the disturbance amplitude 

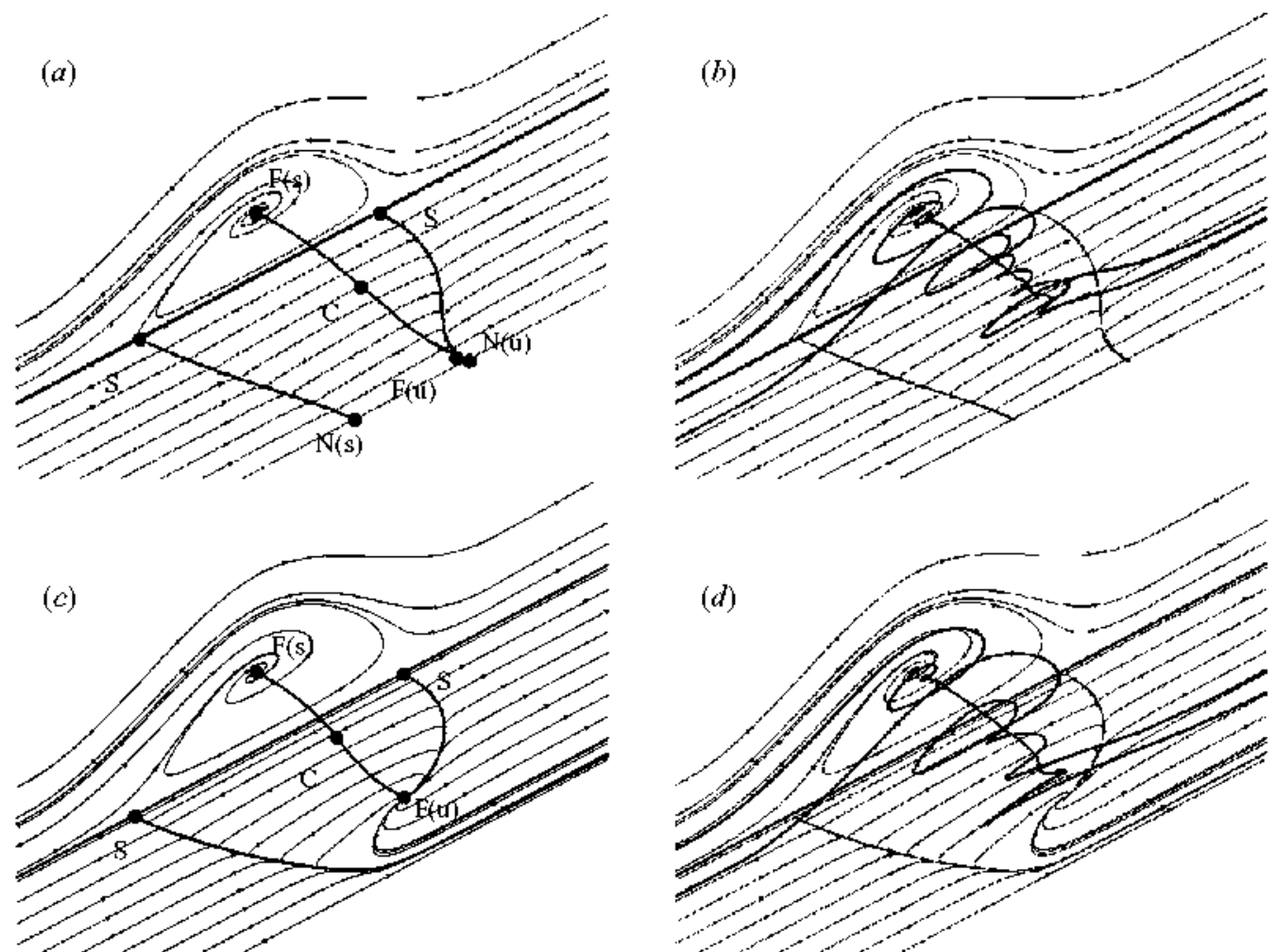

Figure 9. $(a, b)$ Streamlines of the flow reconstruction using the stationary global mode with the lower amplitude. $(c, d)$ Same at higher amplitude. $(a, c)$ Nature of the critical points. $(b, d)$ Three-dimensional streamlines.

exceeds some finite value. To illustrate the new topology a flow reconstruction is done considering a higher amplitude, $\varepsilon=0.2$. Note that these parameter values are indicative of the conditions at which the global eigenvalue problem has been solved presently and would be different at different sets of parameters; of significance here is the qualitatively different nature of the resulting three-dimensional flow field, as opposed to the choice of amplitude parameters in the present linear context. The streamlines trajectories and location of the critical points at the present conditions are shown in the lower part of figure 9. The bifurcation occurs when two critical points, the unstable node in the reattachment line (L3) and the unstable focus (L5) coalesce, giving rise initially to a degenerate node focus ( $\mathrm{H} 35 \mathrm{~d}: Q<0, R>0, S_{1}=0$ ) on the surface streamlines, and afterwards to an unstable focus (H35: $Q<0, R>0, S_{1}>0$ ). For this critical point, the $O(\varepsilon)$ terms are no longer small in (5.10), and the eigendirections are not necessarily parallel to the coordinate planes. The separation stable node L2 disappears, and the other critical points L1, L4 and L6 become H1, H4 and H6. The schematic representation of the critical points is shown in figure $7(b)$. The separation line breaks and folds surrounding the new critical points at the wall: the so called $\mathrm{U}$ shaped separation (Hornung \& Perry 1984; Perry \& Chong 1987; Chong et al. 1990; Dallmann et al. 1997) pattern appears. Counter-rotating swirling flow regions appear symmetrically at both sides of the plane $\Theta=\pi$, in which the surface streamlines are repelled from the foci. The streamlines shown in figure $9(d)$ are strongly reminiscent of the simple U-shaped separation described in Perry \& Chong (1987); even though the 
$(a)$

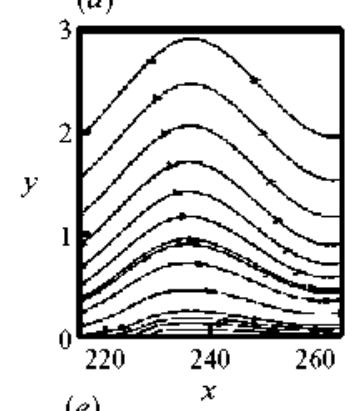

(b)

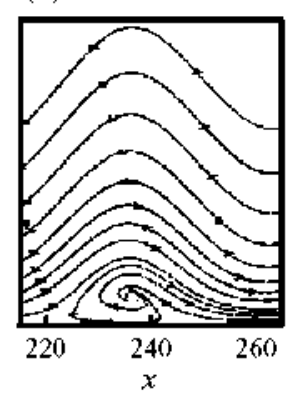

(c)

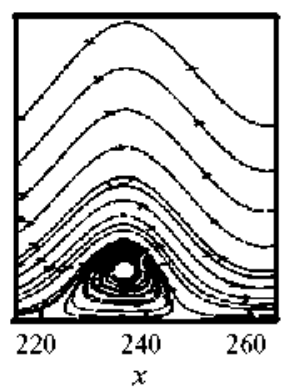

(d)

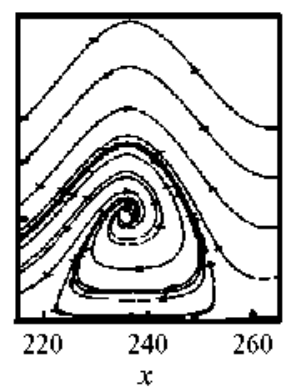

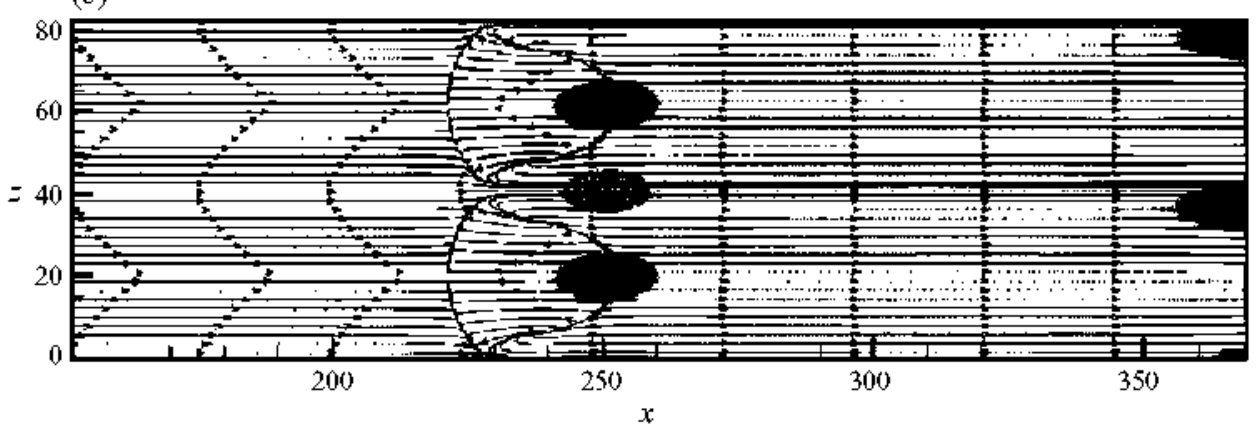

FIgURE 10. Streamlines of the basic flow plus stationary mode with the higher amplitude. ( $a-d$ ) $z$-planes for $\Theta=0(z=0) ; \Theta=0.4 \times \pi(z \approx 9) ; \Theta=\pi / 2(z \approx 10.47)$, and $\Theta=\pi(z \approx 20.94)$. Only a region of the computational domain is shown. (e) Plane $y=0$.

set of critical points describing the topology differs from the present, the trajectory of the fluid particles attracted into the stretching vortex and then repelled transversally is very similar. The surface streamlines along with vertical planes streamlines are shown in figure 10.

\section{Summary and discussion}

It is known from critical point theory that any steady two-dimensional flow, including the LSB boundary layer of interest here, is structurally unstable due to the homogeneity assumption in the third spatial direction and the resulting singular Jacobian matrix. As a consequence, any three-dimensional disturbance will give rise to a three-dimensional flow field, regardless of the amplitude of such disturbance. Here, the structural changes experienced by an incompressible LSB basic flow embedded in a flat-plate boundary layer on account of the presence of its leading stationary global mode (Theofilis et al. 2000) have been studied in detail.

Inverse boundary layer formulation Carter (1975) has been utilized in order to construct steady laminar LSB flows with different amounts of reverse flow. As shown in $\$ 4$, provided the Reynolds number is high enough (as the case is in the present analysis), such model flows are consistent with the full Navier-Stokes equations. BiGlobal instability analysis of the constructed LSB flow models showed that the global mode can be self-excited for a finite range of spanwise wavenumbers; the threshold for self-excitation obtained within the present model is $u_{r e n} \approx 7 \%$. The accuracy of the instability results was verified through DNS employing the well-validated code of Lundbladh et al. (1994). Solutions of the associated adjoint 
eigenvalue problem have also been obtained, since they provide useful information concerning the conditions at which the topologies exerted by the global mode can be realizable. On the one hand, the adjoint eigenfunctions describe the spatial regions at which the global mode is more receptive to external excitation. In the case of the stationary three-dimensional global mode analysed herein the region of highest receptivity is centred at the separation line and extends upstream. On the other hand, the concept of structural sensitivity (Giannetti \& Luchini 2007) shows that the mechanism responsible for the self-excited global mode can be localized in space as the region of highest coupling between the direct and the adjoint eigenfunctions; in the present case this region is highly localized in the vicinity of the recirculation region of the basic state. At recirculation levels above the self-excitation threshold quoted, exponentially amplified perturbations exist in the absence of external forcing. while below this threshold external forcing may continuously excite the same global modes. Consequently, the topological changes discussed herein may be understood as being the result of either the self-excited (unstable) global mode or a continuously excited (stable) such perturbation.

The topological analysis of the composite flow field at low disturbance amplitude shows that the stationary global mode gives rise to a periodic stretching and compression of the spanwise vortex which defines the basic LSB and leads to a spanwise modulation of the separation and reattachment lines. The perturbed separation and reattachment lines are described by a set of connected no-slip (node and saddle) critical points on the wall surface, as seen in figure $9(a, b)$. Identical topologies have been observed experimentally in separation bubbles on a flat plate (Perry \& Fairlie 1975), either by oil-visualization techniques or by particle image velocimetry on iced airfoils (Gurbacki 2003; Jacobs \& Bragg 2006). In the latter experiments two- and three-dimensional castings were used in order to reproduce the ice accretions. Even though the spanwise modulation is much more apparent when the three-dimensional castings were used, it is also present with two-dimensional casting; Jacobs \& Bragg (2006) suggested that the observed three-dimensionality may be a demonstration of the existence of a global mode in the separation bubble.

A new topological bifurcation appears in the reconstructed three-dimensional flow field as the amplitude of the global mode is increased beyond certain thresholds. The separation and reattachment lines break periodically to form multiple symmetric structures; the counter-rotating swirling regions shown in figure $9(c, d)$ appear in the wall streamlines. The resulting flow field shows strong resemblance to the 'stall cells' (also referred to as 'owl-face structures' or 'mushroom structures') observed in experiments on airfoils close to and beyond stall (Winkelmann \& Barlow 1980; Bippes \& Turk 1982; Schewe 2001). A recent study of the appearance of these spanwise periodic structures on the suction side of a stalled airfoil (Rodríguez \& Theofilis 2010 ) has identified stall cells as having their origin in the linear amplification of the global mode of massively separated flow over the airfoil. In the present context the appearance of cellular patterns is also explained by a structural bifurcation which exists beyond certain values of the disturbance amplitude of the global mode of LSB flow, but the structures on the flat plate are essentially different from their counterparts on the airfoil in that no trailing edge is present in the flat plate.

Further work could shed light on two issues. Firstly, three-dimensional DNS are needed in order to quantify the precise role of nonlinearity in the scenario discovered herein. Secondly, and probably more importantly, the relation of the discovered origin of U-separation and spanwise periodic cellular patterns with their counterparts in 
turbulent flow needs to be explored. Both investigations are underway and results will be reported in due course.

This paper is dedicated to the memory of U. Ch. Dallmann. The material is based upon work sponsored by the Air Force Office of Scientific Research, Air Force Material Command, USAF, under Grant number \# FA8655-06-1-3066 to 'nu modelling s.l.', entitled 'Global instabilities in laminar separation bubbles'. The Grant is monitored by Dr D. Smith of AFOSR (originally by Lt. Col. Dr Rhett Jefferies) and Dr S. Surampudi of EOARD. The views and conclusions contained herein are those of the author and should not be interpreted as necessarily representing the official policies or endorsements, either expressed or implied, of the Air Force Office of Scientific Research or the US Government. Computations have been performed on the CeSViMa (www.cesvima.upm.es), MareNostrum (www.bsc.es) and JUGENE (www.fz-juelich.de/jsc/jugene) facilities.

\section{REFERENCES}

Abdessemed, N., Sherwin, S. J. \& Theofilis, V. 2004 On unstable 2 d basic states in low pressure turbine flows at moderate Reynolds numbers. In Thirty-fourth Fluid D vianics Conference and Exhibit. Portland, OR, Paper 2004-2541. AIAA.

Abdessemed. N., Sherwin. S. J. \& Theofilis, V. 2009 Linear instability analysis of low pressure turbine flows. J. Fluid Mech. 628, 57-83.

Åkervik. E.. Hepfffner, J.. Ehrenstein. U. \& Henningson. D. S. 2007 Optimal growth, model reduction and control in a separated boundary-layer flow using global modes. J. Fluid Mech. 579, 305-314.

Allen, T. \& Riley, N. 1995 Absolute and convective instabilities in separation bubbles. Aerosp. J. 99, 439-448.

Barkley, D., Gomes, M. G. M. \& Henderson, R. D. 2002 Three-dimensional instability in a flow over a backward-facing step. J. Fluid Mech. 473, 167-190.

BIPPES, H. \& TURK, M. 1982 Half model testing applied to wing above and below stall. In Recent Contributions to Fluid Mechanics (ed. W. Haase), pp. 22-30. Springer.

Blumen. W. 1970 Shear layer instability of an inviscid compressible fluid. J. Fluid Mech. 40, 769-781.

BoIN, J.-P., RoBinet, J.-C., CORRE, C. \& Denlau, H. 2006 3d steady and unsteady bifurcations in a shock-wave/laminar boundary layer interaction: a numerical study. Theor. Comput. Fhid Dyn. 20, 163-180.

BRILFY, W. R. 1971 A numerical study of laminar separation bubbles using the Navier-Stokes equations. J. Fluid Mech. 47, 713-736.

CARTER, J. E. 1975 Inverse solutions for laminar boundary-layer flows with separation and reattachment. Tech. Rep. TR R-447. NASA.

Ceneci, T. \& CousteIX. J. 2001 Modeling and Computation of Boundary-Layer Flows: Solutions Manual and Computer Programs. Springer.

Cebeci, T., Keller. H. B. \& Williams, P. G. 1979 Separating boundary-layer calculations. J. Comput. Plyys. 31, 363-378.

Chomaz, J. M. 2005 Global instabilities in spatially developing flows: non-normality and nonlinearity. Anmu. Rev. Fluid Mech. 37, 357-392.

Chong, M. S., Perry, A. E. \& Cantwell, B. J. 1990 A general classification of three-dimensional flow fields. Phys. Fluids 2 (5), 765-777.

Crouch, J. D., Garbaruk, A. \& Magidov, D. 2007 Predicting the onset of flow unsteadiness based on global instability. J. Comput. Phys. 224, 924-940.

Dallmann. U. 1982 Topological structures of three-dimensional flow separations. Tech. Rep. DFVLR-IB 221-82 A07.

Dallmann, U. C., Vollmers, H. \& Su, W.-H. 1997 Flow topology and tomography for vortex identification in unsteady and in three-dimensional flows. In IUTAM Symposim on Simulation and Identification of Organized Structures in Flows, pp. $223-238$, Lyngby, Denmark. 
DobrinsKy, A. \& COLLIS, S. S. 2000 Adjoint parabolized stability equations for receptivity prediction. Paper 2000-2651. AIAA.

FAsel, H. \& PostL, D. 2004 Interaction of separation and transtion in boundary layers: direct numerical simulations. In Proceedings of the IUTAM Laminar-Turbulent Symposium $V$ (ed. R. Govindarajan), pp. 71-88. Springer.

Gallaire, F., Marquillie, M. \& Ehrenstein, U. 2007 Three-dimensional transverse instabilities in detached boundary-layers. $J$. Fluid Mech. 571, 221-233.

Giannetri. F. \& Luchini, P. 2007 Structural sensitivity of the first instability of the cylinder wake. J. Finid Mech. 581, 167-197.

Guckenheimer, J. \& Holmes, P. 1983 Nontitear Oscillations. Dynamical Systems and Bifurcations of Vector Fields. Springer.

GURBac:Ki, H. M. 2003 Ice-induced unsteady flowfield effects on airfoil performance. PhD thesis, University of Illinois, Urbana-Champaign, IL.

HAMmond. D. A. \& RedeKopp. L. G. 1998 Local and global instability properties of separation bubbles. Eur. J. Mech. B/Fluids 17, 145-164.

Hill, D. C. 1995 Adjoint systems and their role in the receptivity problem for boundary layers. J. Fluid Mech. 292, 183-204.

Hornung, H. G. \& Perry. A. E. 1984 Some aspects of three-dimensional separation. Part I. Streamsurface bifurcations. Z. Flugwiss. Weltraumforsch. 8, 77-87.

Huer Re, P. \& Monkewitz, P. A. 1985 Absolute and convective instabilities in free shear layers. J. Fluid Mech. 159, 151-168.

Huerre, P. \& Monkewitz, P. A. 1990 Local and global instabilities in spatially developing flows. Amun. Rev. Fluid Mech. 22, 473-537.

JACOBS. J. \& BRAGG, M. B. 2006 Particle image velocimetry measurements of the separation bubble on an iced airfoil. Paper 2006-3646. AIAA.

Kitsios, V. Rodriguez. D., Theofilis, V., OoI, A. \& SoriA. J. 2009 Biglobal stability analysis in curvilinear coordinates of massively separated lifting bodies. J. Comput. Phys. 228, 7181-7196.

LUCHINI, P. 2003 Adjoint methods in transient growth, global instabilities and turbulence control. In Proceedings of the 2nd Symposium on Global Flow Instability and Control, Crete, Greece, Jume 11-13, 2003 (ed. V. Theofilis, T. Colonius \& J.-M. Chomaz). Fundacion General U.P.M. ISBN-13: 978-84-692-6245-0.

Lundbladh, A., Schmid, P., Berlin, S. \& Henningson, D. 1994 Simulation of by-pass transition in spatially evolving flow. In CP-551, pp. 18.1-18.13. AGARD.

MARquet, O., Lombardi. M., Chomaz. J.-M., Sipp, D. \& JACQuin, L. 2009 Direct and adjoint global modes of a recirculation bubble: lift-up and convective non-normalities. J. Fluid Mech. 622, $1-21$.

Marquet, O., SiPP, D., Chomaz, J. M. \& JACQuin, L. 2008 Amplifier and resonator dynamics of a low-reynolds-number recirculation bubble in a global framework. J. Fluid Mech 605, 429-443.

Marquillie, M. \& Ehrenstein, U. 2003 On the onset of nonlinear oscillations in a separating boundary-layer flow. J. Fluid Mech. 490, 169-188.

Michalke, A. 1964 On the inviscid instability of the hyperbolic-tangent velocity profile. J. Finid Mech. 19, 543-556.

Morse, P. M. \& Feshbach, H. 1953 Methods of Theoretical Physics, Parts I, II. MeGraw-Hill.

Perry, A. \& FaIrlie, B. 1975 A study of turbulent boundary-layer separation and reattachment. J. Finid Mech. 69, 657-672.

Perry, A. E. \& Chong, M. S. 1987 A description of eddying motions and flow patterns using critical point concepts. Ammu. Rev. Fluid Mech. 19, 125-156.

Perry, A. E. \& Hornung, H. G. 1984 Some aspects of three-dimensional separation. Part II. Vortex skeletons. Z. Flitgwiss. Weltratmforsch. 8, 155-160.

RIsT, U. \& MAUCHER, U. 2002 Investigations of time-growing instabilities in laminar separation bubbles. Eur. J. Mech. B/Fluids 21, 495-509.

RoBINET, J.-C. 2007 Bifurcations in shock-wave/laminar-boundary-layer interaction: global instability approach. J. Fluid Mech. 579, 85-112.

RODRíGueZ, D. \& TheofiLIs, V. 2008 On instability and structural sensitivity of incompressible laminar separation bubbles in a flat-plate boundary layer. In Thirty-eighth Fluid Dynamics Conference, Seattle, WA. Paper 2008-4148. AIAA. 
Rodríguez, D. \& Theofilis, V. 2009 Massively parallel numerical solution of the biglobal linear instability eigenvalue problem using dense linear algebra. $A I A A J .47(10), 2449-2459$.

Rodríguez, D. \& Theofils, V. 2010 On the birth of stall cells on airfoils. Theor. Comput. Fluid Dyn. Special Issue on Global Flow Instability and Control (in pressi (doi:10.1007/s00162-0100193-7).

SAlwen, H. \& Grosch, C. 1981 The continuous spectrum of the Orr-Sommerfeld equation. Part 2. Eigenfunction expansions. J. Fluid Mech. 104, 445-465.

Schewe, G. 2001 Reynolds-number effects in flow around more-or-less bluff bodies. J. Wind Engng Ind. Aerodyn. 89, 1267-1289.

Schmid, P. \& Henningson, D. 2001 Stability and Transition in Shear Flows. Springer.

SPALART, P. R. 1988 Direct numerical study of leading-edge contamination. In CP-438 Fluid Dynamics of Three-Dimensional Turbulent Shear Flows and Transition, Pp. 5.1-5.13. Cesme, Turkey. AGARD.

Theofilis, V. 2000 Global linear instability in laminar separated boundary layer flow. In Proceedings of the IUTAM Laminar-Turbulent Symposium V (ed. H. Fasel \& W. Saric), pp. 663-668. Springer. Sedona, AZ.

THEofiLIs, V. 2003 Advances in global linear instability analysis of nonparallel and three-dimensional flows. Prog. Aerosp. Sci. 39, 249-315.

Theofilis, V. 2009 The role of instability theory in flow control. In Findamentals and Applications of Modern Flow Control. (ed. R. D. Joslin \& D. Miller), AIAA Progress in Aeronautics and Astronautics, vol. 231, pP. 73-116. AIAA.

Theofilis, V., BARKLeY, D. \& SHERwin. S. J. 2002 Spectral/hp element technology for flow instability and control. Aeronaut. J. 106, 619-625.

Theofilis, V., Fedorov, A. Obrist, D. \& Dallmann, U. C. 2003 The extended GörtlerHämmerlin model for linear instability of three-dimensional incompressible swept attachmentline boundary layer flow. J. Fluid Mech. 487, 271-313.

Theofilis, V.. Hein, S. \& Dallmann, U. 2000 On the origins of unsteadiness and threedimensionality in a laminar separation bubble. Phit. Trans. R. Soc. Lowd. A 358, 3229-3324.

Tumin, A. 2003 Multimode decomposition of spatially growing perturbations in a two-dimensional boundary layer. Phys. Flidds 15 (9), 2525-2540.

VoRobiefF, P. \& Rockwell, D. 1996 Wavelet filtering for topological decomposition of flow fields. Intl J. Imaging Syst. Technol. 7, 211-214.

Winkelmann, A. \& Barlow, B. 1980 Flowfield model for a rectangular planform wing beyond stall. $A I A A$ J. 8, 1006-1008. 